\title{
Proton-Coupled Electron Transfer in a Series of Ruthenium-Linked Tyrosines with Internal Bases: Evaluation of a Tunneling Model for Experimental Temperature-Dependent Kinetics
}

\author{
Todd F. Markle, ${ }^{\dagger}$ Ming-Tian Zhang, ${ }^{\ddagger}$ Marie-Pierre Santoni, ${ }^{\S}$ Linus O. Johannissen, \\ and Leif Hammarström*
}

Department of Chemistry - Ångström Laboratory, Uppsala University, P.O. Box 523, S-75120 Uppsala, Sweden

Supporting Information

ABSTRACT: Photoinitiated proton-coupled electron transfer (PCET) kinetics has been investigated in a series of four modified tyrosines linked to a ruthenium photosensitizer in acetonitrile, with each tyrosine bearing an internal hydrogen bond to a covalently linked pyridine or benzimidazole base. After correcting for differences in driving force, it is found that the intrinsic PCET rate constant still varies by 2 orders of magnitude. The differences in rates, as well as the magnitude of the kinetic isotope effect $\left(\mathrm{KIE}=k_{\mathrm{H}} / k_{\mathrm{D}}\right)$, both generally correlate with DFT calculated proton donor-acceptor dis-
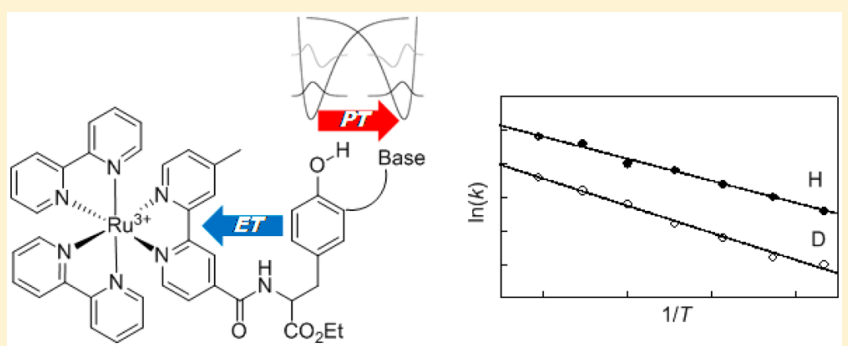
tances. An Arrhenius analysis of temperature dependent data shows that the difference in reactivity arises primarily from differences in activation energies. We use this kinetic data to evaluate a commonly employed theoretical model for proton tunneling which includes a harmonic distribution of proton donor-acceptor distances due to vibrational motions of the molecule. Applying this model to the experimental data yields the conclusion that donor-acceptor compression is more facile in the compounds with shorter PT distance; however, this is contrary to independent calculations for the same compounds. This discrepancy is likely because the assumption in the model of Morse-shaped proton potential energy surfaces is inappropriate for (strongly) hydrogenbonded systems. These results question the general applicability of this model. The results also suggest that a correlation of rate vs proton tunneling distance for the series of compounds is complicated by a concomitant variation of other relevant parameters.

\section{INTRODUCTION}

Concerted electron-proton transfer (CEPT) - the transfer of electrons and protons in a single kinetic step-is recognized as a potential mechanistic pathway in a number of enzymatic systems and artificial catalytic systems. ${ }^{1-5}$ These processes are a subset of hydrogen transfer reactions (i.e., proton transfer, hydrogen atom transfer, hydride transfer $)^{6}$ which have intrigued chemists for decades, in part because of the role of nuclear tunneling. Renewed interest in these processes has come from the push to develop catalytic systems for the generation of "solar fuels", e.g., the splitting of water into $\mathrm{O}_{2}$ and $\mathrm{H}_{2}$ (eq la), which will necessarily involve a number of proton coupled electron transfers. This is also true for the key biological water splitting equivalent (eq $1 \mathrm{~b}$ ), photosystem II, for which important recent progress has been made in unraveling the mechanistic details. ${ }^{7-9}$

$$
\begin{aligned}
& 2 \mathrm{H}_{2} \mathrm{O} \rightleftharpoons \mathrm{O}_{2}+2 \mathrm{H}_{2} \\
& 2 \mathrm{H}_{2} \mathrm{O} \rightleftharpoons \mathrm{O}_{2}+4 \mathrm{H}^{+}+4 \mathrm{e}^{-}
\end{aligned}
$$

Model systems have been useful for understanding of the key parameters defining CEPT reactivity. Phenols have been of particular interest in that regard because of the prevalence of tyrosine residues in electron transfer pathways in radical enzymes. A number of groups have investigated phenol oxidations in model systems to probe the key parameters which define CEPT reactivity. ${ }^{10-24}$ One parameter in particular that has received attention is the proton transfer distance. The increased mass of the proton relative to that of an electron makes proton tunneling much more strongly dependent on the distance. This strong distance dependence also means that molecular vibrations and motions accessible at room temperature can have significant effects on tunneling rates by modulating the proton transfer distance. Such motions are implicated in enzymatic catalysis. $^{25-34}$ Modeling these reactions is challenging in part due to the extreme sensitivity to dynamic fluctuations in geometry on both the reactant and product electronic surfaces. One approach is computational modeling of reaction rates using a semiclassical vibronic treatment. ${ }^{5,35}$

In this work, we will use a model based on work by Kuznetsov and Ulstrup, which is a special case of the more general semiclassical vibronic treatment, and which will be described in the next section. We apply this to model experimental temperaturedependent rates and kinetic isotope effects for a series of synthetic complexes. The modeling is robust, in the sense that it

Received: June 10, 2016

Revised: August 4, 2016

Published: August 4, 2016 

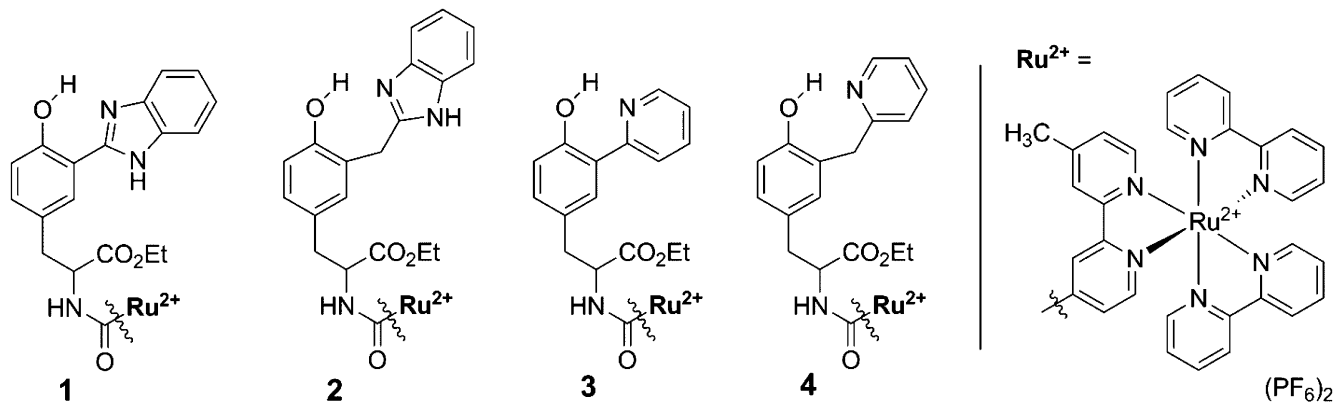

Figure 1. Ruthenium linked tyrosine-base compounds, 1-4, studied.

includes only a few free model parameters while using an extensive set of experimentally determined parameters. It includes nuclear quantum effects in a tractable manner using assumptions about the proton's chemical environment and the motions which modulate the proton transfer distance. One goal of our work is to test this theoretical treatment of CEPT reactivity and its assumptions, and to thereby shed light onto the relative importance of various parameters. The present work follows on a preliminary report of photoinitiated CEPT in a series of compounds, 1-4 (Figure 1), where electron transfer from the modified tyrosine to photogenerated $\mathrm{Ru}^{\mathrm{III}}$ occurs with concomitant intramolecular proton transfer to the attached pyridyl or imidazolyl base. Previous studies have established, on the basis of thermodynamic-kinetic arguments, that the proton and electron are transferred in a single concerted kinetic step via CEPT. ${ }^{12}$ The CEPT reaction can safely be assumed to be nonadiabatic, as the electronic coupling for long-range electron transfer through saturated bonds is expected to be small $\left(V_{\mathrm{ET}}<\right.$ $\left.25 \mathrm{~cm}^{-1}\right) .{ }^{35}$ This is also supported by our modeling results below.

The role of proton transfer distance on CEPT has been discussed in a number of studies of model phenol systems, with widely differing conclusions. A detailed computational study found that decreased distance was a key factor in favoring proton transfer to phosphate buffer over bulk water in a rheniumtyrosine system. ${ }^{36-38}$ In a series of papers by Mayer and coworkers, a simple correlation of rates and distance was, in general, not observed. ${ }^{39-43}$ For example, in a report from 2006, ${ }^{39}$ three phenols bearing either an amino, pyridine, or imidazole basic substituent were found to react with rates which vary by a factor of ca. $10^{2}$ after extrapolation to similar driving forces, but these rates do not correlate with the crystallographic proton donoracceptor distance, $d_{\mathrm{ON}}$. Instead, it was noted that compounds with stronger hydrogen bonding interactions displayed higher rate constants. This was supported by a follow-up study of two phenol-pyridines (analogues of $\mathbf{3}$ and $\mathbf{4}$ ) where it was found that the presence of a methylene spacer between the phenol and a pyridine base resulted in a much longer $d_{\mathrm{ON}}$ but similar CEPT reactivity as observed in an amino analogue. ${ }^{40}$ A related study from some of us compared two analogous phenol-carboxylates, which also differed by the presence of a methylene unit. ${ }^{18}$ In these carboxylates, the proton donor-acceptor distance was quite similar, but the effect on kinetics was nevertheless comparable to that observed in the pyridines, and the key effect was determined to be differences in the motions which modulate the proton transfer distance (see below). In contrast, a later study from Mayer and co-workers found that an amino-phenol in which $d_{\mathrm{ON}}$ is ca. $0.15 \AA$ longer resulted in rates that were much more similar, differing only by a factor of ca. $3-10{ }^{43}$ Further, the differences in rates were dependent on $\Delta G^{\circ}$, and there was a counterintuitive decrease in the kinetic isotope effect $\left(k_{\mathrm{H}} / k_{\mathrm{D}}, \mathrm{KIE}\right)$ with an increase in $d_{\mathrm{ON}}$. In contrast, a first communication of the PCET reactions of compounds $\mathbf{1 - 4}$ showed the more intuitively satisfying result of a strong decrease in PCET rate with increasing distance. $^{18}$

The present work adds to this discussion and describes in more detail the CEPT kinetics of compounds $\mathbf{1 - 4}$ in acetonitrile, including the temperature dependence of the KIE. We use the data to evaluate the model of Kuznetsov and Ulstrup, described in detail below, in the hopes of gaining further insights into the relative importance of proton transfer distance, tunneling, proton donor-acceptor motions, and hydrogen bonding on CEPT kinetics.

Introduction to the Theoretical Model. CEPT kinetics can be described by a model resembling that of Marcus theory for outer-sphere electron transfer eq 2 , which relates the rate constant $k_{\mathrm{CEPT}}$ to the thermodynamic driving force for the CEPT process $\Delta G^{\circ}$, reorganization energies for solute and solvent $\lambda$, and a term which describes the coupling between product and reactant surfaces at the transition state, $V_{\mathrm{CEPT}}{ }^{44}$ Given the quantum nature of the transferring proton, vibrationally excited states must be considered for the reactants and products ( $\mu$ and $\nu$, respectively) (eq 3). The energies of the proton vibrational states, given by eq 5 for a quantum Morse oscillator, will affect $k_{\mathrm{CEPT}}$ via the population of the reactant state, $P_{\mu}$, and by modulating the driving force, $\Delta E_{\mathrm{vib}, \mu \nu}$. However, the vibrational states of the proton will also affect the coupling terms $V_{\mu \nu}$, given by eq $6{ }^{45}$ where $V_{\mathrm{ET}}$ is the electronic coupling term and the bracketed term, $V_{\mathrm{PT}}$, is the spatial overlap between the reactant and product wave functions. This overlap should decrease exponentially as the tunneling distance, $r_{x}$, is increased around a reference distance, $r_{0}$ (eq 7). ${ }^{24,46,58} V_{\mathrm{CEPT}}$ will also be dependent on isotope as more massive isotopes will have more localized wave functions; however, the energies of more diffuse vibrational excited states become more accessible with heavier isotopes.

$$
\begin{aligned}
& k_{\mathrm{CEPT}}=\frac{1}{2 \pi \hbar} V_{\mathrm{CEPT}}^{2} \sqrt[2]{\frac{4 \pi^{3}}{\lambda k_{\mathrm{B}} T}} \exp \left[-\frac{\left(\Delta G^{\circ}+\lambda\right)^{2}}{4 \lambda k_{\mathrm{B}} T}\right] \\
& k_{\mathrm{CEPT}}=\sum_{\mu} P_{\mu} \sum_{\nu} \frac{1}{2 \pi \hbar}\left|V_{\mu \nu}\right|^{2} \sqrt{\frac{4 \pi^{3}}{\lambda k_{\mathrm{B}} T}} \exp \left[\frac{-\Delta G_{\mu \nu}^{\ddagger}}{k_{\mathrm{B}} T}\right] \\
& \Delta G_{\mu \nu}^{\ddagger}=\frac{\left(\Delta G^{\circ}+\Delta E_{\mu \nu}^{\mathrm{vib}}+\lambda\right)^{2}}{4 \lambda} \\
& E_{n}^{\mathrm{vib}}=(n+1 / 2) \hbar \omega-\frac{((n+1 / 2) \hbar \omega)^{2}}{4 D_{\mathrm{e}}} \\
& V_{\mu \nu} \approx V_{\mathrm{ET}}\left\langle\phi_{\mu} \mid \phi_{\nu}\right\rangle
\end{aligned}
$$


Here, we employ a modification of the model of Kuznetsov and Ulstrup, ${ }^{47}$ previously implemented by Knapp et al., ${ }^{48}$ Meyer et al., ${ }^{49}$ Johannissen et al., ${ }^{30}$ and Hay et al. ${ }^{31}$ This is a thermal averaging procedure for a nonadiabatic vibronic rate constant expression, resulting in eqs 7 and 8 below. A key simplifying assumption in this approach is that the hydrogen bond potential energy surface is modeled as a Morse potential, which allows for the proton wave functions to be calculated analytically. Vibrations which modulate the proton donor-acceptor distance $\left(d_{\mathrm{ON}}\right)$ will also change the tunneling distance $r_{x}$ (Figure 2).
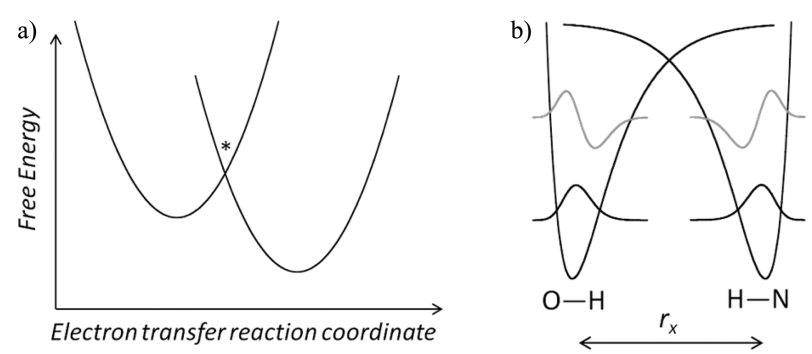

Figure 2. Schematic representation of the Kuznetsov-Ulstrup model for PCET. (a) The electronic portion is treated in a Marcus fashion. (b) Proton tunneling at the transition state (* in panel a) with Morse curves for reactant and product hydrogen bonds. The distance between the bottom of the wells is modulated from its equilibrium value $r_{0}$ by vibrations of the heavy nuclei; the projection of these normal modes on the $\mathrm{O}-\mathrm{N}$ coordinate is treated as one effective harmonic oscillator with force constant $\kappa_{x}$.

These motions are included as a classical harmonic oscillator, with force constant $\kappa_{x}$, and the energy required to distort from the equilibrium distance, $r_{0}$, is $E_{x}={ }^{1} /{ }_{2} \Delta r^{2} \kappa_{x}$. ${ }^{18}$

With the assumption of a Boltzmann distribution of $r_{x}$, the nuclear wave function overlap at temperature $T$ is then the integral of the overlap terms for all possible values of $\Delta r$, weighted by their respective population:

$$
\left\langle\phi_{\mu} \mid \phi_{\nu}\right\rangle^{2}=\int_{r=0}^{\infty}\left[\left\langle\phi_{\mu} \mid \phi_{\nu}\right\rangle_{r}{ }^{2} \frac{\exp \left(-E_{x} / k_{\mathrm{B}} T\right)}{\int_{\Delta r=0}^{\infty} \exp \left(-E_{x} / k_{\mathrm{B}} T\right)}\right]
$$

Combining eqs 3, 6, and 7 gives the full rate expression

$$
\begin{aligned}
k_{\mathrm{CEPT}}= & \sum_{\mu} P_{\mu} \sum_{\nu} \frac{1}{2 \pi \hbar}\left|V_{\mathrm{ET}}\right|^{2} \sqrt{\frac{4 \pi^{3}}{\lambda k_{\mathrm{B}} T}} \exp \left[\frac{-\Delta G_{\mu \nu}^{+}}{k_{\mathrm{B}} T}\right] \\
& \times \int_{r=0}^{\infty}\left[\left\langle\phi_{\mu} \mid \phi_{\nu}\right\rangle_{r}{ }^{2} \frac{\exp \left(-E_{x} / k_{\mathrm{B}} T\right)}{\int_{\Delta r=0}^{\infty} \exp \left(-E_{x} / k_{\mathrm{B}} T\right)}\right]
\end{aligned}
$$

With the further assumptions that $\lambda$ and $V_{\mathrm{ET}}$ remain constant for all vibrational levels and $\Delta r$ values, there are only three variable parameters in the model which are modified during the fitting, while there are four independent experimental observables to which the model is fitted. These observables are the driving force $\left(\Delta H^{\circ}\right.$ and $\left.\Delta S^{\circ}\right), k_{\mathrm{CEPT}}, E_{\mathrm{a}}, \Delta E_{\mathrm{a}}$ (the difference in activation energy between the two isotopes), and the kinetic isotope effect $\left(k_{\mathrm{H}} / k_{\mathrm{D}}\right)$ at $298 \mathrm{~K}$, while the independent model parameters are $V_{\mathrm{ET}}, \lambda$, and $\kappa_{x}$. Additional parameters that define the wave functions for the transferring proton/deuteron ( $\nu_{\mathrm{XH}}$ and $D_{\mathrm{e}}$ of the hydrogen bond potential) and the equilibrium tunneling distance $r_{0}$ are determined using DFT and are not modified to fit the data.

The fitting procedure is as follows: (i) The force constant for the donor-acceptor vibrations, $\kappa_{x}$, is modified to fit the experimental KIE at $298 \mathrm{~K}$. (ii) The Marcus reorganization energy, $\lambda$, is modified to fit the experimental $E_{\mathrm{a}}$. (iii) The electronic coupling, $V_{\mathrm{ET}}$, is modified to fit $k_{\mathrm{H}}$ at $298 \mathrm{~K}$.

\section{RESULTS AND DISCUSSION}

Structural Characterization. Compounds 1-4 (Figure 1) in which the tyrosine-base unit is linked to a $\left[\mathrm{Ru}(\mathrm{bpy})_{3}\right]^{2+}$ photosensitizer have been prepared as $\mathrm{PF}_{6}$ salts, as described in the Supporting Information of ref 19. The saturated linkage between the modified tyrosine residue and ruthenium-bipyridine units makes their structures and electronics fairly independent; therefore, analogous phenol-base compounds, lacking the photosensitizer and instead bearing tert-butyl groups ortho and para to the phenolic oxygen (compounds $\mathbf{1}^{\prime}-4^{\prime}$, see the Supporting Information), serve as useful reference systems.

The proton transfer distance and hydrogen bond geometry are expected to be important parameters for the evaluation of CEPT reactivity. Crystallographic structures have been previously reported for $\mathbf{1}^{\prime},{ }^{41,50,51} \mathbf{3}^{\prime},{ }^{39}$ and $\mathbf{4}^{\prime},{ }^{16,40}$ and a structure for $\mathbf{2}^{\prime}$ was reported in the preliminary communication on this work. ${ }^{19}$ In all four compounds, there exists an $\mathrm{OH} \cdots \mathrm{N}$ intramolecular hydrogen bond. However, the hydrogen bond geometry, e.g., the distance between the proton donor (phenolic oxygen) and acceptor (basic nitrogen) $\left(d_{\mathrm{ON}}\right.$, Table 1$)$, can be strongly affected by crystal packing forces. This point is illustrated in compound $\mathbf{1}^{\prime}$ for which two structures with otherwise similar hydrogen bonding have been reported with a total of four independent molecules. ${ }^{41,50}$ The $d_{\mathrm{ON}}$ values in these four molecules vary from 2.533(3) to 2.6087(19) A. Similar variations in $d_{\mathrm{ON}}$ have been observed in crystal structures of related compounds. ${ }^{39,42}$ Because $d_{\mathrm{ON}}$ can be strongly influenced by crystal packing, DFT calculated structures are very useful and will be described below.

\begin{tabular}{|c|c|c|c|c|c|c|c|c|}
\hline & $d_{\mathrm{ON}}, \mathrm{X}_{\text {-ray }}^{b}$ & $d_{\mathrm{ON}}(\mathrm{R})^{c}$ & $d_{\mathrm{ON}}(\mathrm{P})^{c}$ & $\theta_{\mathrm{R}}^{c}$ & $\theta_{\mathrm{P}}^{c}$ & $r_{0}^{c, d}$ & $\delta_{\mathrm{OH}}^{h}$ & $\nu_{\mathrm{OH}}{ }^{c}$ \\
\hline 1 & $\begin{array}{l}2.539(2), 2.609(2)^{e} \\
2.533(3), 2.547(2)^{f} 2.739(2)^{g}\end{array}$ & 2.610 & 2.662 & 20.4 & 36.2 & 0.892 & 13.04 & 3161 \\
\hline 2 & $2.752(5)^{h}$ & 2.717 & 2.771 & 11.0 & 28.5 & 0.895 & 10.19 & 3188 \\
\hline 3 & $2.561(3), 2.567(3), 2.573(3)^{i}$ & 2.561 & 2.600 & 19.1 & 25.2 & 0.685 & 14.13 & 3003 \\
\hline 4 & $2.691(1),{ }^{j} 2.696(1)^{k}$ & 2.711 & 2.724 & 11.2 & 19.2 & 0.769 & 10.71 & 3134 \\
\hline
\end{tabular}
Compounds $\mathbf{1}^{\prime}$ and $\mathbf{3}^{\prime}$ are essentially planar, with very similar $d_{\mathrm{ON}}$

Table 1. Experimental and DFT Computed Structural and Spectroscopic Properties ${ }^{a}$

${ }^{a}$ Distances in $\AA$, angles in degrees, $\delta_{\mathrm{OH}}$ is the experimental NMR chemical shift of the phenolic proton in dried $\mathrm{CD}_{3} \mathrm{CN}$ in ppm, $\nu_{\mathrm{OH}}$ is the DFT calculated IR stretching frequency in $\mathrm{cm}^{-1},(\mathrm{R})$ and $(\mathrm{P})$ indicate values for the optimized geometries reactant (reduced) and oxidized product, $\theta_{\mathrm{R}}=\angle_{\mathrm{HON}}, \theta_{\mathrm{P}}=\angle_{\mathrm{HNO}} \cdot{ }^{b} \mathrm{X}$-ray, crystallographic values are listed for all available independent molecules for compounds $1^{\prime}-4^{\prime}$. ${ }^{c} \mathrm{Calculated}$ at $\mathrm{B} 3 \mathrm{LYP} / 6-311++\mathrm{G}(\mathrm{d}, \mathrm{p})$ with the polarizable continuum model of $\mathrm{CH}_{3} \mathrm{CN}$ solvent. ${ }^{d}$ Estimated equilibrium proton transfer distance; see text. ${ }^{e}$ Reference 50. ${ }^{f}$ Reference $41 .{ }^{g}$ Reference $51 .{ }^{h}$ Reference $19 .{ }^{i}$ Reference $39 .{ }^{j}$ Reference $40 .{ }^{k}$ Reference 16. 

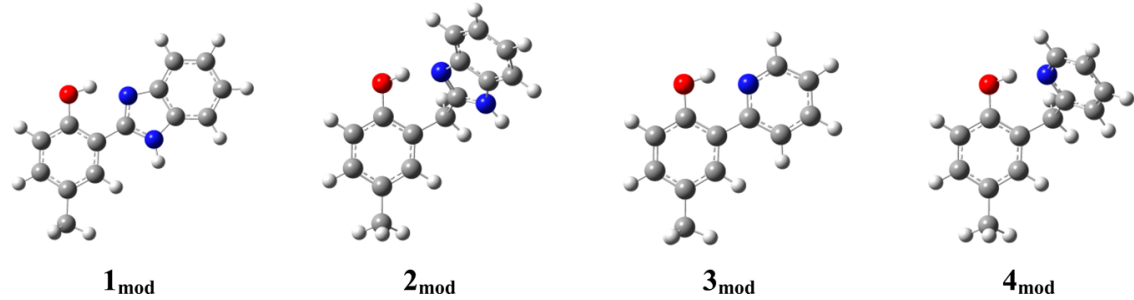

Figure 3. Optimized structures for the truncated model compounds of 1-4. Note, crystallographically characterized analogues from the literature, $\mathbf{1}^{\prime}-4^{\prime}$, each bear tert-butyl groups ortho and para to the phenolic hydroxyl group.

(Table 1 ). In compounds $\mathbf{2}^{\prime}$ and $\mathbf{4}^{\prime}$ where the phenol ring and heterocyclic base are separated by a methylene unit and no longer locked into a rigid $\pi$ system, the base twists up out of the plane of the phenol and results in longer $d_{\mathrm{ON}}$ (see DFT calculated structures, Figure 3 ). Compound $2^{\prime}$ has the longest $d_{\mathrm{ON}}$ of the series; however, in the crystal structure of $2^{\prime}$, the basic nitrogen of the benzimidazole displays bifurcated hydrogen bonding interactions with both the phenolic $\mathrm{OH}$ as well as the $\mathrm{N}-\mathrm{H}$ proton of a neighboring benzimidazole (with intra- and intermolecular hydrogen bond donor-acceptor distances of 2.752(5) and 3.009(5) $\AA$, respectively). This bifurcation of the hydrogen bond in the structure of $2^{\prime}$ is likely a strong contributor to the increased crystallographic $d_{\mathrm{ON}}$ by twisting the basic nitrogen away from the phenol: the torsion angle $d \angle_{\mathrm{C}-\mathrm{C}=\mathrm{N} \cdots \mathrm{O}}$ is $32.2(4)^{\circ}$ for $2^{\prime}$ compared to $6.2(1)^{\circ}$ for $4^{\prime}$. Simple rotation of the benzimidazole about the $\mathrm{H}_{2} \mathrm{C}$-benzimidazole bond in $2^{\prime}$ such that $d \angle_{\mathrm{C}-\mathrm{C}=\mathrm{N} \cdots \mathrm{O}}$ matches that of $4^{\prime}$ decreases $d_{\mathrm{ON}}$ by $0.16 \AA$, making it $0.1 \AA$ shorter in $2^{\prime}$ vs $4^{\prime}$. A very similar bifurcation is present in one of the three reported structures for $\mathbf{1}^{\prime}$, and results in a significantly longer $d_{\mathrm{ON}}$ of 2.739(2) $\AA^{51}$ These bifurcated hydrogen bonds in the crystal structures are not likely relevant to the situation in dilute $\mathrm{MeCN}$ solution, where the solvent can act as a hydrogen bond acceptor but not as a donor. This situation is analogous to a structure obtained by Wenger and co-workers for an analogue of $4^{\prime}$ which displayed intermolecular phenol-pyridine hydrogen bonded chains in the crystal (and, therefore, an extremely long intramolecular $d_{\mathrm{ON}}=4.396(2) \AA$, cf. intermolecular hydrogen bond distance $d_{\mathrm{ON}}=2.740(2) \AA$ ); however, in solution, it was concluded that the intramolecularly hydrogen bonded conformation was dominant via ${ }^{1} \mathrm{H}$ NMR studies. ${ }^{16}$

DFT calculated geometries avoid complications that arise from crystal packing forces. Geometries have been optimized for analogues of 1-4, in which the amino acid tethered $\mathrm{Ru}$ photosensitizer has been truncated to a methyl group para to the phenolic oxygen. Unless otherwise specified, the geometries reported have been calculated using B3LYP/6-311++G(d,p) and a polarizable continuum model (PCM) of acetonitrile as implemented in Gaussian 09. ${ }^{52}$ For compounds 1-4, use of a smaller basis set, e.g., 6-31G(d,p), produced only minor quantitative differences in geometries and relative energies (see, for example, Table S5); however, diffuse functions are necessary for the anionic phenol-carboxylate systems. These calculations predict $d_{\mathrm{ON}}$ which span $0.156 \AA$, from $2.561 \AA$ (3) to $2.717 \AA$ (2). The DFT structures generally have slightly longer $d_{\mathrm{ON}}$ compared to the experimental structures, with the exception of $\mathbf{2}$ where the bifurcated hydrogen bonding in the crystal of $2^{\prime}$ likely leads to an increased $d_{\mathrm{ON}}$. While crystallographic values of $d_{\mathrm{ON}}$ yield the trend $3^{\prime} \sim 1^{\prime}<4^{\prime}<2^{\prime}$, the DFT values are $3<1<4 \sim 2$. Inclusion of an explicit $\mathrm{MeCN}$ solvent molecule hydrogenbonded to the imidazole $\mathrm{N}-\mathrm{H}$ of $\mathbf{1}$ and $\mathbf{2}$ yields $d_{\mathrm{ON}}$ values which are only slightly shorter (by $\lesssim 0.01 \AA$ ). The DFT calculations also allow for more detailed information on the location of the phenolic proton than is available from crystallographic structures. As noted previously, ${ }^{40}$ the seven-membered rings in 2 and 4 allow for a more linear hydrogen bond $\left(\theta_{\mathrm{R}}=11^{\circ}\right.$ for both $\mathbf{2}^{\prime}$ and $4^{\prime}$ vs 20 and $19^{\circ}$ for $\mathbf{1}^{\prime}$ and $\left.3^{\prime}\right)$. These structures offer an interesting comparison to an analogous pair of tyrosinecarboxylates which also differ by a methylene between the phenol and base. ${ }^{17,18}$ DFT structures have been reported for those two compounds, and while the compound with the methylene again allows for a more linear hydrogen bond, the proton donoracceptor distances are more similar to each other: $d_{\mathrm{OO}}=2.52$ and $2.59 \AA$ and $\theta_{\mathrm{R}}=16.8$ and $8.3^{\circ}$ for the conjugated salicylic acid derivative (SA) and the nonconjugated 2-hydroxyphenylacetic acid (PA), respectively. ${ }^{18}$ This is likely due to the fact that the negative charge on the basic carboxylate in each compound is more important than any conjugation effect. ${ }^{53,54}$

Estimations of the Hydrogen Bond Strength. DFT calculations also allow for the direct estimation of the hydrogen bond strength. One computational measure of $\mathrm{H}$-bond strength is the difference in energy between $\mathrm{H}$-bonded and non-H-bonded conformations when the hydroxyl group is rotated, with all other atoms fixed at the hydrogen bonded structure. At the PCM-B3LYP/6-31G(d,p) level, this yields $\mathrm{H}$-bond strengths of $58-66 \mathrm{~kJ} \mathrm{~mol}^{-1}$ following the trend $\mathbf{2}=\mathbf{4}<\mathbf{1}<\mathbf{3}$ (see Table S2 for values). There is a much smaller difference between $\mathbf{1}$ and $\mathbf{2}$ compared to $\mathbf{3}$ and $\mathbf{4}$, as was also seen for the computed vibrational wavenumbers, $\nu_{\mathrm{OH}}$; see below. Alternatively, the hydrogen bond strength can be calculated as the energy difference between fully optimized "open" and "closed" forms (with the hydroxyl group pointed away from, or toward, the base). This treatment has the advantage of being a direct calculation of the thermodynamic strength of the hydrogen bond $\left(\Delta H_{\mathrm{HB}}\right)$, although the non-hydrogen-bonded species is not present in sufficient concentrations to be observed experimentally (e.g., by solution IR spectra $\left.{ }^{39,40}\right)$. This treatment yields values of $\Delta H_{\mathrm{HB}}$ which span a greater range (from 21 to $44 \mathrm{~kJ} \mathrm{~mol}^{-1}$ ), and the trend is somewhat different, $2<4<1 \sim 3$.

The variation in hydrogen bonding also manifests itself in spectral changes. It was noted previously for $3^{\prime}$ and $4^{\prime}$ that the presence of the $\mathrm{CH}_{2}$ spacer leads to a large effect on the $\mathrm{O}-\mathrm{H}$ stretching mode, $\nu_{\mathrm{OH}}$, in the IR spectra ${ }^{40}$ ascribed to resonanceassisted hydrogen bonding (RAHB). ${ }^{53,54}$ This is seen also at this level of theory (Table 1$)$, with $\nu_{\mathrm{OH}}$ of $3\left(3003 \mathrm{~cm}^{-1}\right)$ being $130 \mathrm{~cm}^{-1}$ lower than that of $4\left(3134 \mathrm{~cm}^{-1}\right)$. However, the calculated $\nu_{\mathrm{OH}}$ values for $\mathbf{1}$ and $\mathbf{2}$ are both higher, and more similar to each other ( 3161 and $3188 \mathrm{~cm}^{-1}$ ). RAHB has been criticized, with the argument that the "resonance assistance" is actually a consequence of the $\mathrm{H}$-bond donor and acceptor being forced closer together as a result of the rigid $\pi$ system. ${ }^{55}$ The present results may support those claims in that the difference in $\nu_{\mathrm{OH}}$ is less dramatic in $\mathbf{1}$ and $\mathbf{2}$ vs $\mathbf{3}$ and $\mathbf{4}$. Perhaps this is an effect of the 
five-membered ring of the imidazole, which directs the basic lone pair of the nitrogen away from the hydroxyl group.

${ }^{1} \mathrm{H}$ NMR provides a convenient experimental probe for the chemical environment of the transferring proton under conditions similar to those of the kinetic experiments (spectra were collected in $\mathrm{CD}_{3} \mathrm{CN}$ ). Chemical shifts of the phenolic protons are shifted downfield, as is known for hydrogen bonded systems. The chemical shift $\left(\delta_{\mathrm{OH}}\right)$ in $\mathrm{CD}_{3} \mathrm{CN}$ varies from 10.19 to $14.13 \mathrm{ppm}$ following the trend $\mathbf{2}<\mathbf{4}<\mathbf{1}<\mathbf{3}$, Table 1 , following approximately the trend of calculated hydrogen bond strengths above. The chemical shifts for $\mathbf{1 - 4}$ are shifted somewhat upfield (by $0.44-0.7 \mathrm{ppm}$ ) from those reported previously for $\mathbf{1}^{\prime}, \mathbf{3}^{\prime}$, and $4^{\prime}$ which bear bulky di-tert-butyl groups on the phenol ring.

Kinetics Measurements. The PCET rate constants were obtained in acetonitrile using a flash-quench method with transient absorption detection described previously. ${ }^{17,56}$ The $\mathrm{Ru}$ compound was excited by a $5 \mathrm{~ns}$ laser pulse at $460 \mathrm{~nm}$, producing the MLCT excited state: ${ }^{*} \mathrm{Ru}^{\mathrm{II}}$. The excited state was oxidatively quenched by methyl viologen $\left(\mathrm{MV}^{2+}\right)$ yielding $\mathrm{Ru}$ III within $150 \mathrm{~ns}$ according to the bimolecular reaction (9a):

$$
\begin{aligned}
& \mathrm{MV}^{2+}+{ }^{*} \mathrm{Ru}^{\mathrm{II}} \mathrm{TyrOH} \cdots \mathrm{B} \rightarrow \mathrm{MV}^{\bullet+}+\mathrm{Ru}^{\mathrm{III}} \mathrm{TyrOH} \cdots \mathrm{B} \\
& \mathrm{Ru}^{\mathrm{III}} \mathrm{TyrOH} \cdots \mathrm{B} \rightarrow \mathrm{Ru}^{\mathrm{II}} \mathrm{TyrO}^{\bullet} \cdots \mathrm{HB}^{+} \\
& \mathrm{Ru}^{\mathrm{III}} \mathrm{TyrOH} \cdots \mathrm{B}+\mathrm{MV}^{\bullet+} \rightarrow \mathrm{Ru}^{\mathrm{II}} \mathrm{TyrOH} \cdots \mathrm{B}+\mathrm{MV}^{2+}
\end{aligned}
$$

The first quenching reaction step (9a) was monitored by the emission decay of $* \mathrm{Ru}^{\mathrm{II}}$ at $600 \mathrm{~nm}$ and by the buildup of transient absorption signals at 390 and $600 \mathrm{~nm}$, as a signature of $\mathrm{MV}^{+\bullet}$. The subsequent intramolecular PCET reaction from the TyrOH...B unit to $\mathrm{Ru}^{\mathrm{III}}$ (9b) was monitored by the recovery of the $\mathrm{Ru}^{\mathrm{II}}$ signal at $450 \mathrm{~nm}$. Examples of kinetic traces and fits are given in Figure S1. When the PCET reaction was sufficiently rapid to ignore recombination of $\mathrm{MV}^{+\bullet}$ with $\mathrm{Ru}^{\mathrm{III}}$ (9c), the traces were fitted with a single exponential function, with rate constant $k_{\text {PCET }}$. When recombination was non-negligible on the PCET time scale, kinetic data were fitted with eq 10, where the second order recombination rate constant $\left(k_{\text {rec }} ;\right.$ eq 9c) was determined independently from the $600 \mathrm{~nm}$ decay traces of the $\mathrm{MV}^{\bullet+}$ (see the Supporting Information for a derivation of eq 10).

$$
\frac{\left[\mathrm{Ru}^{\mathrm{III}}\right]}{\left[\mathrm{Ru}^{\mathrm{III}}\right]_{0}}=\frac{\mathrm{e}^{-k_{\mathrm{PCET}} t}}{1+k_{\mathrm{rec}} t\left[\mathrm{MV}^{\bullet+}\right]_{0}}
$$

The room temperature flash-quench experiments reported in ref 19 yield rates for CEPT which vary by a factor of 6.8 from 0.93 to $6.30 \times 10^{4} \mathrm{~s}^{-1}$; however, $\Delta G^{\circ}$, determined via electrochemical measurements, ${ }^{19}$ varies such that $K_{\text {eq }}$ spans ca. 4 orders of magnitude (Table 2). When $\ln k_{\mathrm{H}}$ is plotted vs $\ln K_{\text {eq }}$ (Figure $4 \mathrm{a}$ ), we see a strong but shallow correlation, with more downhill reactions displaying lower rate constants, in direct contrast to the prediction of eq 2. This dependence of rates upon driving force is opposite to that predicted from eq 2 and eq 8 in the so-called Marcus normal region of relatively mild driving forces, when $-\Delta G^{\circ}<\lambda,^{44}$ as is the case here. The apparent contradiction can be explained by the fact that the compounds with the shorter proton transfer distance (1 and 3) have the lower driving force. However, using Marcus theory to extrapolate the rates in the various systems to $\Delta G^{\circ}=0$ (in the limit where $\Delta G^{\circ} \ll \lambda^{57}$ ) gives $\ln k_{\mathrm{H}}+\Delta G^{\circ} / 2 R T$, which we here denote as $\ln k_{0}$. This allows for comparisons of reactivity among 1-4 at the same driving force, assuming that the variation of the activation energy and reaction
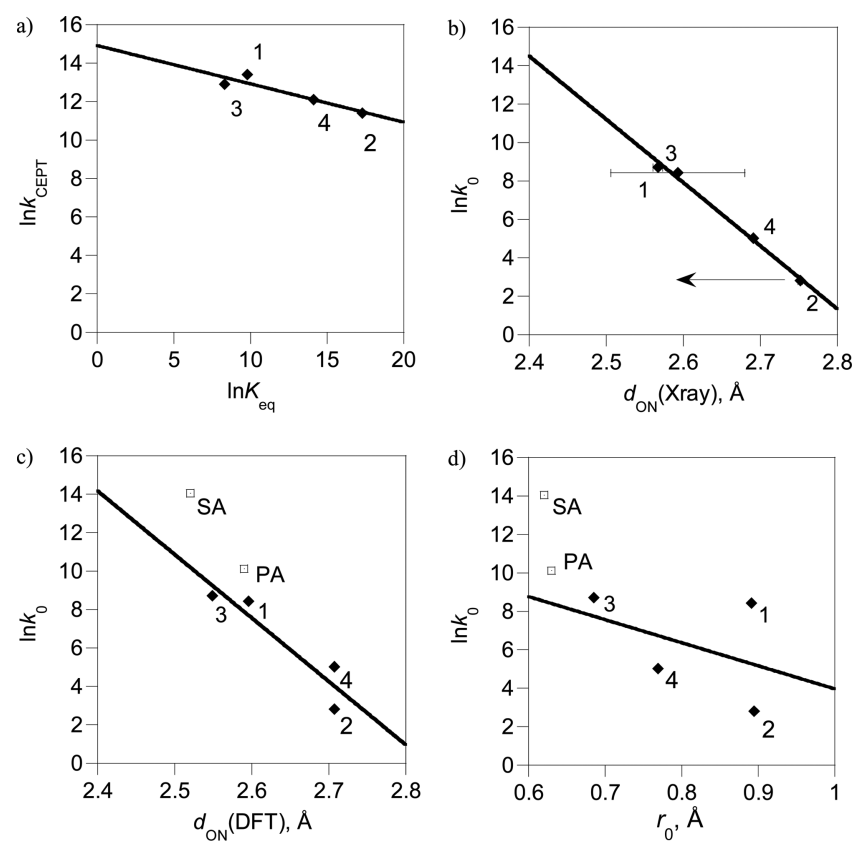

Figure 4. (a) Plot of $\ln k_{\mathrm{CEPT}}$ vs $\ln K_{\mathrm{eq}}$. (b) Plot of $\ln k_{0}$ vs the average crystallographic $d_{\mathrm{ON}}$; error bars are the larger of the crystallographic esd, or one standard deviation of the averaged values in Table 1. The horizontal arrow on the point corresponding to compound 4 indicates that this crystallographic distance is likely an overestimate of the distance in solution (see text). (c) Plot of $\ln k_{0}$ vs $d_{\mathrm{ON}}$ from DFT calculations. (d) Plot of $\ln k_{0}$ vs DFT calculated proton transfer distance, $r_{0}$ (see Figure 6). Black diamonds correspond to compounds 1-4, and open squares correspond to Ru-linked tyrosine-carboxylates (kinetics collected in aqueous solution) from ref 18 . Lines are linear fits to the points for $\mathbf{1 - 4}$.

free energy, $\Delta \Delta G^{*} / \Delta \Delta G^{\circ}$ or $\alpha$, is predicted to be $1 / 2$. This treatment has been used previously for similar reactions. ${ }^{13,19,42}$

Trends in Experimental Kinetic Data. Before we model our data, we examine some trends. Various parameters change across this series of compounds such as the electrochemical potential and $\mathrm{p} K_{\mathrm{a}}$ of the phenol and base, the geometry and rigidity of the hydrogen bond, and the chemical environment of the transferring proton, and further complications arise because these parameters may be correlated to one another to different degrees. However, with these factors in mind, here we focus on the proton transfer distance, and examine whether its effect on the rate is dominating over that of variation in other parameters. The trend in $\ln k_{0}, 2<\mathbf{4}<\mathbf{1} \sim 3$, closely mirrors the trends in crystallographic $d_{\mathrm{ON}}$, and a plot of $\ln k_{0}$ versus the crystallographic $d_{\mathrm{ON}}$ values (Figure $4 \mathrm{~b}$ ) yields a strong linear correlation with a slope of $33 \AA^{-1}$; this is somewhat larger than the value of $27 \AA^{-1}$ reported in ref 16 due to the revised average $d_{\mathrm{ON}}$ for $\mathbf{1}^{\prime}$ (see above). Using $d_{\mathrm{ON}}$ as a proxy for the proton tunneling distance, and with the assumption that $\lambda$ stays constant across the series, eq 2 can be rewritten as eq 11, when the driving force is small compared to $\lambda$. Therefore, the slope of the best fit line in Figure $4 \mathrm{~b}$ gives $\beta$, and this value is similar to that predicted from relatively simple proton tunneling models. ${ }^{24,58-60}$ However, this correlation depends heavily on the structure for $2^{\prime}$ with the bifurcated hydrogen bond. If $\ln k_{0}$ is plotted vs $d_{\mathrm{ON}}$ values from DFT calculations (Figure $4 \mathrm{c}), \beta$ is similar in magnitude $\left(33 \AA^{-1}\right)$ yet more scatter is apparent $\left(R^{2}=0.86\right.$ vs 0.97$)$, suggesting a role for other parameters. For example, compounds $\mathbf{2}$ and $\mathbf{4}$ which have very similar $d_{\mathrm{ON}}$ values (within $0.01 \AA$ ) have intrinsic rates, $k_{0}$, which differ by a factor of 9 , while compounds 1 and 3 which 
have very similar $k_{0}$ differ in $d_{\mathrm{ON}}$ by ca. $0.05 \AA$. If instead the estimated proton tunneling distance $r_{0}$ is used, which also depends on hydrogen bond angles (see Figure 6), the correlation with $\ln k_{0}$ becomes worse (Figure $4 \mathrm{~d}, R^{2}=0.18$ ). This is primarily because of compound 1 which is predicted to have an $r_{0}$ distance very similar to that of $\mathbf{2}$. If this point is neglected, a strong linear correlation is achieved with $\beta=27 \AA^{-1}$.

$$
\ln k_{\mathrm{CEPT}}+\frac{\Delta G^{\circ}}{2 R T}=-\beta d_{\mathrm{ON}}+\text { constant }
$$

It is interesting to note that if kinetic data from the analogous ruthenium-linked tyrosine-carboxylate compounds, ${ }^{18}$ which also differ by a methylene unit between the phenol and the basic moiety, are included in this analysis, the correlation falls roughly in line with the data for $\mathbf{1 - 4}$ (Figure $4 \mathrm{c}$ and $\mathrm{d}$ ). This is in spite of the fact that that study was carried out in water as solvent. The computation of $\ln k_{0}$ for these two compounds $\left(\ln k_{0}=14\right.$ and 10) is less certain due to uncertainties in $\Delta G^{\circ}$; however, the kinetic effect of the methylene unit is very similar to that observed in the two imidazoles and pyridines. Plots of $\ln k_{0}$ vs DFT calculated proton donor-acceptor distance yield a strong correlation with $\beta=46\left(R^{2}=0.85\right)$ when the two tyrosinecarboxylate complexes from ref 18 are also included (denoted SA and PA in Figure $4 \mathrm{c}$ and d). Plotting $\ln k_{0}$ vs $r_{0}$ gives $\beta=24$ $\left(R^{2}=0.56\right)$ (or a very good correlation with $\beta=36, R^{2}=0.87$, excluding the data point for 1). However, again, significant scatter is apparent; e.g., the difference in $k_{0}$ between the two tyrosine-carboxylates where $r_{0}$ differs by only $0.01 \AA$ is greater than that between 3 and 4 , which have $r_{0}$ which differ by $0.084 \AA$.

Kinetics were also determined for $\mathbf{1 - 4}$ as a function of temperature, $T=283-313 \mathrm{~K}$, to determine Arrhenius parameters (Figure 5, Table 2). Parameters obtained from computations and simulations of the experimental data, as described below, are given in the adjacent Tables 3-4 to facilitate comparison of relevant trends. The trend in activation energy
$\left(E_{\mathrm{a}}\right)$ values roughly follows the trend in $d_{\mathrm{ON}}, \mathbf{1}<3<4<2$, and likewise, although the values for the prefactor $A$ for 1,3 , and 4 are within experimental uncertainty of each other $\left(A=\mathrm{ca} .10^{10.5}\right)$, the value is largest for compound $2\left(A=10^{11.7}\right)$, which has the longest $d_{\mathrm{ON}}$ and lowest $\ln k_{0}$ of the series. This is perhaps surprising because it is expected that an increased $A$ factor corresponds to increased tunneling from reactant to product surfaces and should decrease with increased proton-tunneling distance. However, because the reaction likely involves thermal motions which modulate this distance and possibly vibrationally excited reactant states (see above), the tunneling terms are expected to be temperature dependent (eq 8), and the reaction may no longer be tunneling-limited in the limit of high temperature. It is noteworthy that $E_{\mathrm{a}}$ values are higher for compounds 2 and 4, despite the fact that CEPT is more downhill in those compounds. These results clearly indicate that the variation in reactivity in this series is manifested in differences in energetic terms $\left(E_{\mathrm{a}}\right)$, whether they arise from proton donor-acceptor motions $\left(\kappa_{x}\right)$ and excited proton vibronic states as included in the Ulstrup-Kuznetsov model or from classical reorganization energies $(\lambda)$, and not from temperature independent electronic and proton coupling terms.

The phenolic proton is readily exchanged with deuterium in the presence of $\mathrm{D}_{2} \mathrm{O}$; therefore, kinetic isotope effects were obtained from flash-quench experiments performed using $0.75 \% \mathrm{D}_{2} \mathrm{O}$ in acetonitrile which is a large excess relative to Ru-linked tyrosine (ca. $10 \mu \mathrm{M}$ ). Control experiments were carried out in the presence of the same amount of $\mathrm{H}_{2} \mathrm{O}$, and the ratio of these rates $\left(\mathrm{H}_{2} \mathrm{O} / \mathrm{D}_{2} \mathrm{O}\right)$ yields the kinetic isotope effect $\left(k_{\mathrm{H}} / k_{\mathrm{D}}\right)$. These range from 1.42 to 4.02 following the trend $\mathbf{1}<\mathbf{3}<\mathbf{2}=\mathbf{4}$, Table 2 . These primary kinetic isotope effects, together with kinetic and thermodynamic arguments, ${ }^{19}$ are key indications of the CEPT mechanism in these systems. The difference in KIE between $\mathbf{3}$ and $\mathbf{4}$ is comparable to, if somewhat less than, what was reported by Markle and Mayer in bimolecular a) 1

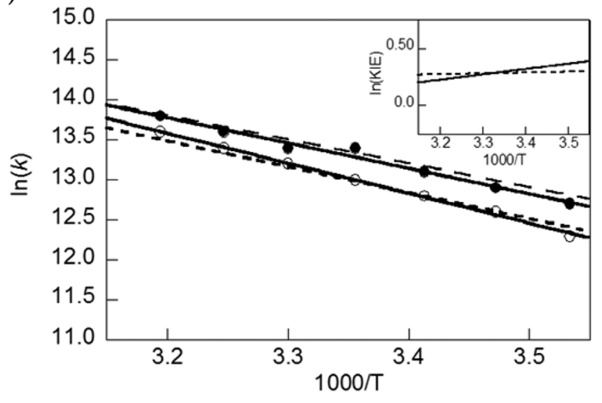

c) 3

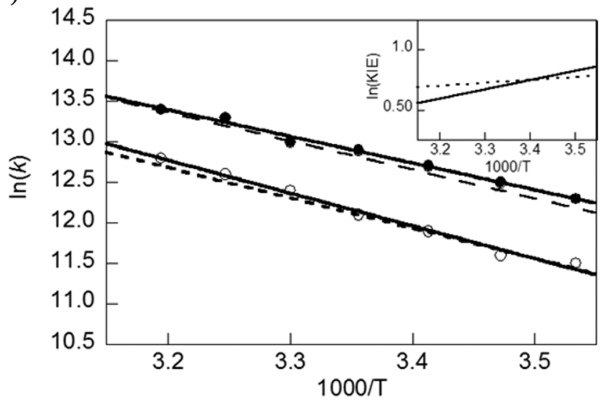

b) 2

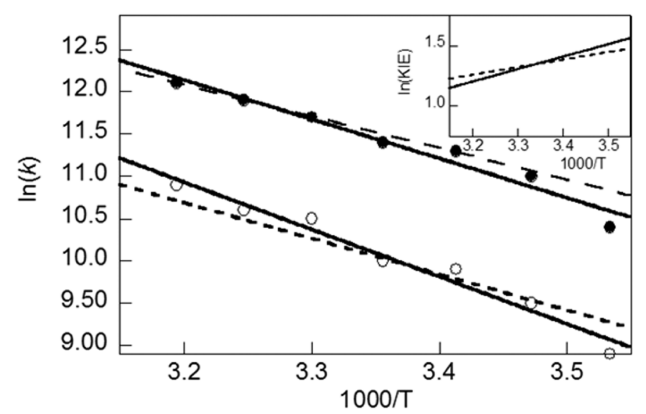

d) 4

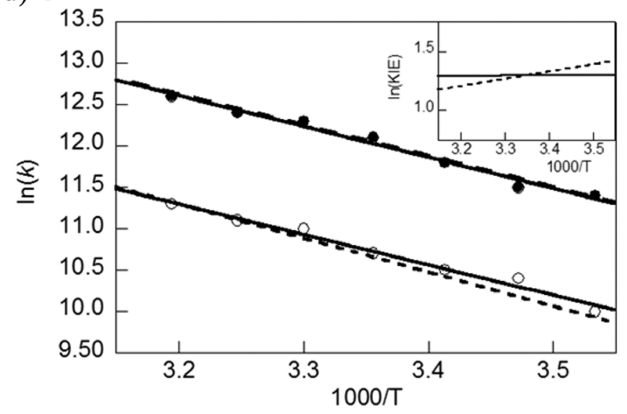

Figure 5. Variation of $k_{\mathrm{H}}$ (filled circles) and $k_{\mathrm{D}}$ (open circles) with temperature for CEPT in 1-4. Solid lines are Arrhenius fits. Dashed lines are the temperature dependences predicted from the modeling studies (see text). The rate constant values are also given in Table S1. 
Table 2. Experimental Parameters for CEPT Kinetics

\begin{tabular}{|c|c|c|c|c|c|c|c|c|}
\hline & $\Delta G^{\circ a}(\mathrm{eV})$ & $k_{\mathrm{H}}{ }^{a} 10^{5} \mathrm{~s}^{-1}$ & $\ln k_{0}^{a, b}$ & $k_{\mathrm{H}} / k_{\mathrm{D}}{ }^{a}$ & $E_{\mathrm{a}}\left(\mathrm{kJ} \mathrm{mol}^{-1}\right)$ & $\ln A$ & $E_{\mathrm{a}}^{\mathrm{D}}-E_{\mathrm{a}}^{\mathrm{H}}\left(\mathrm{kJ} \mathrm{mol}^{-1}\right)$ & $\ln \left(A^{\mathrm{H}} / A^{\mathrm{D}}\right)$ \\
\hline 1 & -0.252 & $6.30 \pm 0.03$ & 8.4 & 1.42 & $25.8 \pm 1.0$ & $23.7 \pm 0.4$ & $3.87 \pm 1.16$ & $-1.3 \pm 0.5$ \\
\hline 2 & -0.443 & $0.93 \pm 0.01$ & 2.8 & 4.01 & $38.6 \pm 3.5$ & $27.0 \pm 1.4$ & $8.60 \pm 5.26$ & $-2.1 \pm 2.1$ \\
\hline 3 & -0.212 & $3.81 \pm 0.01$ & 8.7 & 2.16 & $27.7 \pm 0.5$ & $24.0 \pm 0.2$ & $6.48 \pm 1.75$ & $-1.9 \pm 0.7$ \\
\hline 4 & -0.363 & $1.78 \pm 0.01$ & 5.0 & 4.02 & $30.6 \pm 1.2$ & $24.4 \pm 0.5$ & $0.10 \pm 1.69$ & $1.3 \pm 0.7$ \\
\hline
\end{tabular}

Table 3. DFT Calculated Parameters for the Hydrogen Bond Potentials of $1-4^{a}$

$\begin{array}{cccccc} & r_{0}{ }^{b} & \nu_{\mathrm{OH}}(\mathrm{R}) & \nu_{\mathrm{NH}}(\mathrm{P}) & D_{\mathrm{e}}(\mathrm{R}) & D_{\mathrm{e}}(\mathrm{P}) \\ \mathbf{1} & 0.892 & 3161 & 3463 & 173 & 391 \\ \mathbf{2} & 0.895 & 3188 & 3432 & 174 & 371 \\ \mathbf{3} & 0.685 & 3003 & 3174 & 140 & 264 \\ \mathbf{4} & 0.769 & 3134 & 3159 & 173 & 254\end{array}$

${ }^{a} \mathrm{IR}$ stretching frequencies $\left(\nu_{\mathrm{XH}}\right)$ are in $\mathrm{cm}^{-1}$, and dissociation energies $\left(D_{\mathrm{e}}\right)$ are in $\mathrm{kJ} \mathrm{mol}^{-1} \cdot{ }^{b}$ Estimated equilibrium proton transfer distance; see text (repeated from Table 1).

CEPT oxidations of $3^{\prime}$ and $4^{\prime}$ by $\left[\mathrm{Fe}\left(5,5^{\prime}-\mathrm{Me}_{2} \mathrm{bpy}\right)_{3}\right]^{3+}$ (which were $2.5 \pm 0.6$ and $5.9 \pm 0.8) .{ }^{39,40}$ Plotting $\ln k_{0, \mathrm{D}}$ vs measurements of distance produces similar trends as those observed for the proteo reactions but with steeper slopes; e.g., $\ln k_{0, \mathrm{D}}$ vs $d_{\text {ON }}\left(\right.$ DFT) yields $\beta_{\mathrm{D}}=40 \AA^{-1}$ (compared to $33 \AA^{-1}$ for $\mathrm{H}$ ).

The KIE was determined as a function of temperature, and Arrhenius analysis gives $\Delta E_{\mathrm{a}}\left(E_{\mathrm{a}, \mathrm{D}}-E_{\mathrm{a}, \mathrm{H}}\right)$ values which range from $0.1 \pm 1.7$ to $8.6 \pm 5.3 \mathrm{~kJ} \mathrm{~mol}^{-1}$ (Figure 5, Table 2). For 1-3, the activation energy is larger $\left(\Delta E_{\mathrm{a}}>0\right)$ and the prefactor is higher $\left(\ln \left(A^{\mathrm{H}} / A^{\mathrm{D}}\right)<0\right)$ for the deuterated analogue. This is consistent with vibrationally assisted PCET in the UlstrupKuznetsov model, where the vibrational energy of the effective mode that modulates the proton donor-acceptor distance $d_{\mathrm{ON}}$ is moderately smaller than the thermal energy, $h v<k T$, but not so much smaller that the semiclassical limit is reached, where the reaction occurs without tunneling. ${ }^{47,48}$ Compound 4, with the longest $d_{\mathrm{ON}}$, shows instead Arrhenius parameters which fall within the limits that can be described with semiclassical theory. ${ }^{61}$ This may be an indication that the reactions of 4 is in a temperature regime where tunneling contribution is less significant, ${ }^{62}$ or involve an increased role of vibrationally excited reactant states. ${ }^{63}$ This will be discussed further below after modeling of the data. However, we note here that the parameters for $\mathbf{4}$ are very susceptible to experimental uncertainty, due to the limited range in temperature and rate constants. For example, if the data points taken at $288 \mathrm{~K}$ are neglected, the $\Delta E_{\mathrm{a}}$ value for 4 increases to $2.0 \pm 1.2 \mathrm{~kJ} \mathrm{~mol}^{-1}$. Significant variation of KIE with temperature was previously determined by stopped flow for the closely related bimolecular reaction of $4^{\prime}$ with $\mathrm{Fe}\left(\mathrm{Me}_{2} \mathrm{bpy}\right)_{3}{ }^{3+}$ $\left(\Delta E_{\mathrm{a}}=9.6 \pm 5.0 \mathrm{~kJ} \mathrm{~mol}^{-1}\right.$ and $\left.\log \left(A_{\mathrm{H}} / A_{\mathrm{D}}\right)=-2.3 \pm 1.8\right) .{ }^{40}$
On the other hand, small values of $\Delta E_{a}$ comparable to that determined for 4 , have been reported for bimolecular CEPT reactions of phenol-amines. ${ }^{43}$

Modeling of Kinetic Data. Computational Inputs. Along with the experimentally determined kinetic parameters $\left(k_{\mathrm{H}}\right.$ and $k_{\mathrm{D}}$ at $298 \mathrm{~K}, E_{\mathrm{a}}$ and $\left.\Delta E_{\mathrm{a}}\right)$ and thermodynamic driving force, several parameters required for fitting the data for compounds 1-4 to eq 8 are derived from DFT calculations. The equilibrium proton tunneling distance, $r_{0}$, discussed above, is one of these parameters.

The proton donor-acceptor distance, $d_{\mathrm{ON}}$, is only a proxy for the parameter which should actually influence CEPT reactivity, which is the proton tunneling distance, $r$, i.e., the reactantproduct well separation at the Marcus transition state. Given the quantum nature of the proton, this distance may be difficult to rigorously define, and it is complicated by the fact that the tunneling distance requires knowledge of the proton position in both reactant and product species, before and after the double tunneling CEPT event. While the majority of the CEPT reactivity in solution occurs from transition state conformations that are distorted from the equilibrium geometry as indicated by the significant energetic barriers observed, the proton tunneling distance at the optimized geometry, $r_{0}$ (Figure 6), should give

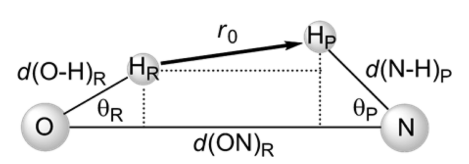

Figure 6. Schematic depiction of the hydrogen bond in reactant and product states and tunneling distance, $r_{0}$.

insight into relative proton tunneling distances. In order to estimate $r_{0}$, some consideration of the geometry of the oxidized species is necessary. DFT calculated $d_{\mathrm{ON}}$ values in the oxidized products $d_{\mathrm{ON}}(\mathrm{P})$ are somewhat longer than the neutral reactants $d_{\mathrm{ON}}(\mathrm{P})(2.600(3)-2.771(2) \AA)$ but follow a similar trend: $3<1$ $<\mathbf{4}<\mathbf{2}$ (Table 1). DFT also predicts the hydrogen bond in the cation product to be more bent compared to the corresponding neutral species. However, the trend in the linearity of the $\mathrm{H}$-bond $\left(\theta_{\mathrm{P}}=\angle_{\mathrm{HNO}}\right)$ is different: $4<3 \sim 2<\mathbf{1}$, with the two pyridine compounds having more linear hydrogen bonds than the

Table 4. Kinetic Parameters Derived from Numerical Modeling of Experimental Kinetic Data and Calculated $\lambda_{\mathrm{i}, \mathrm{DFT}}$ Values

\begin{tabular}{|c|c|c|c|c|c|c|c|}
\hline & $\lambda^{a}\left(\mathrm{~kJ} \mathrm{~mol}{ }^{-1}\right)$ & $\lambda_{\mathrm{i} \mathrm{DFT}}{ }^{b}$ & $V_{\mathrm{ET}}^{a}\left(\mathrm{~cm}^{-1}\right)$ & $\kappa_{x}\left(\mathrm{~J} \mathrm{~m}^{-2}\right)$ & $\Delta E_{\mathrm{a}}\left(\mathrm{kJ} \mathrm{mol}^{-1}\right)$ & $\partial \ln k_{\mathrm{H}} / \partial r_{0}$ & $\partial \ln k_{\mathrm{D}} / \partial r_{0}$ \\
\hline 1 & 72.5 & 40 & 1.8 & 3.9 & 0.603 & 7.4 & 7.7 \\
\hline 2 & 85.1 & 44 & 6.5 & 16.1 & 5.22 & 22.2 & 25.2 \\
\hline 3 & 60.4 & 33 & 1.8 & 13.2 & 2.1 & 14.6 & 16.5 \\
\hline 4 & 73.4 & 48 & 3.6 & 21.2 & 5.1 & 21.7 & 25.3 \\
\hline $\mathrm{Ru}-\mathrm{SA}^{c}$ & 101 & 37 & 69 & 11.9 & 1.51 & & \\
\hline $\mathrm{Ru}-\mathrm{PA}^{c}$ & 128 & 41 & 72 & 21.2 & 2.68 & & \\
\hline
\end{tabular}

${ }^{a}$ The magnitudes of $\lambda$ and $V_{\mathrm{ET}}$ are strongly dependent on the roughly estimated values of $\Delta S^{\circ}$; see the Supporting Information. ${ }^{b}$ Calculated inner sphere contribution to the reorganization energy, eq $12 .{ }^{c}$ From ref 18 , with $\Delta S^{\circ}=+0.05 \mathrm{~kJ} \mathrm{~mol}^{-1} \mathrm{~K}^{-1}$. 
benzimidazoles. Using the DFT calculated structures, the equilibrium proton transfer distance $r_{0}$ has been estimated following a previous report, ${ }^{18}$ using $d_{\mathrm{ON}}$ for the reactant species, and $\theta_{\mathrm{R}}$ and $\theta_{\mathrm{P}}$ (Figure 6, Table 1 ). The $0.21 \AA$ range in $r_{0}$ values is somewhat larger than the span of $d_{\mathrm{ON}}$ values. However, because of the variation in the hydrogen bond angles in the series, $d_{\mathrm{ON}}$ and $r_{0}$ are not well correlated. $r_{0}$ values follow the trend $3<\mathbf{4}<\mathbf{1}$ $\sim 2$ with the two benzimidazole compounds, which differ in $d_{\mathrm{ON}}$ by $0.11 \AA$ having essentially the same $r_{0}$ (within $0.01 \AA$ ). Also, comparing the conjugated benzimidazole and pyridine compounds 1 and 3 where the $d_{\mathrm{ON}}$ values differ by $0.05 \AA$, the $r_{0}$ values differ more than $0.2 \AA$. This is primarily due to the hydrogen bond angle in the oxidized species: $\theta_{\mathrm{P}}=36.2^{\circ}(1)$ vs $25.2^{\circ}(3)$.

In addition, parameters are needed to describe the shape of the $\mathrm{H}$-bond potential. The $\mathrm{H}$-bond is modeled as the Morse potential. This is a significant simplification in these hydrogen bonded systems, as will be discussed below, but allows for the analytic solution of the anharmonic proton vibrational wave functions for reactant $(\mathrm{OH}$ bound phenol) and product $(\mathrm{NH}$ bound phenoxyl radical) species. The Morse potentials are defined by the frequency, $\nu_{\mathrm{XH}}$, here derived from DFT normalmode analysis, and the dissociation energy, $D_{\mathrm{e}}$, taken from potential energy surface scans along $d_{\mathrm{XH}}$ for the reduced $(\mathrm{OH})$ and oxidized ( $\mathrm{NH}$ ) forms (Table 3 ).

Results from Numerical Modeling. In this section, we discuss the parameters which come out of the numerical modeling of the temperature-dependent kinetic data in Figure $4, \kappa_{x}, \lambda, V_{\mathrm{ET}}$, and $\Delta E_{\mathrm{a}}$; the values are summarized in Table 4 (cf. eqs 2-7). A number of similarities are observed between the results for 1-4 compared to the previous results of the tyrosine-carboxylates. ${ }^{18}$ The presence of the methylene spacer in $\mathbf{2}$ and $\mathbf{4}$ compared to 1 and 3 results in an increase in $\lambda$ and a higher force constant for donor-acceptor vibrations, both of which contribute to the decreased $k_{0}$ and increased KIE. However, 2 and 4 also show an increase in $V_{\mathrm{ET}}$ relative to $\mathbf{1}$ and 3 . The increase in $k_{0}$ caused by changes in $V_{\mathrm{ET}}$ essentially cancels out the effect of the increase in $\lambda$; e.g., comparing compounds 2 and 3 which have the lowest and highest values of $k_{0}, \lambda$ for compound 2 is $25 \mathrm{~kJ} \mathrm{~mol}^{-1}$ higher than that of 3 , which corresponds to a decrease in $k_{0}$ by a factor of ca. 12 at room temperature. However, the corresponding increase in $V_{\mathrm{ET}}$ leads to an increase in $k_{0}$ by nearly the same amount $\left(V_{\mathrm{ET}}(2)^{2} / V_{\mathrm{ET}}(3)^{2} \sim 14\right)$. In fact, for compound 1 where the combined effect of $\lambda$ and $V_{\mathrm{ET}}$ is largest, these terms only contribute to a decrease by a factor of ca. 3 compared to $2-4$.

The trends in $\lambda$ and $V_{\mathrm{ET}}$ in general seem to be reasonable. The values of $\lambda$, the total reorganization energy, obtained from the modeling studies range from 60 to $85 \mathrm{~kJ} \mathrm{~mol}^{-1}$ and follow the trend $3<\mathbf{1} \sim \mathbf{4}<\mathbf{2}$. Inner-sphere reorganization energies have also been calculated for the phenolic moiety in 1-4 independently using Nelsen's four-point method adapted to include the proton transfer in $\mathrm{CEPT}^{18,23,64-66}$

$$
\lambda_{\mathrm{i}, \mathrm{DFT}}=1 / 2\left[\left(E_{\mathrm{R}(\mathrm{P})}-E_{\mathrm{R}}\right)+\left(E_{\mathrm{P}(\mathrm{R})}-E_{\mathrm{P}}\right)\right]
$$

where $E_{\mathrm{R}}$ and $E_{\mathrm{P}}$ are the potential energies of the reactant and product at their respective optimized structures. $E_{\mathrm{R}(\mathrm{P})}$ is the energy of the reactant with all nontransferring atoms fixed at the position optimized for the product; likewise, $E_{\mathrm{P}(\mathrm{R})}$ is the energy of the oxidized product with all atoms except the transferring proton in the reactant conformation. These calculations utilize gas phase B3LYP/6-311++G(d,p) energies calculated at geometries optimized at the same level of theory with a continuum model of $\mathrm{MeCN}$ solvent. As has previously been discussed, ${ }^{45}$ the inner-sphere reorganizations for $\left[\mathrm{Ru}(\mathrm{bpy})_{3}\right]^{3+/ 2+}$ are negligible; ${ }^{67}$ therefore, these values are analogous to the inner sphere contribution to $\lambda$ obtained from the modeling of the experimental kinetic data. For 1-4, $\lambda_{\mathrm{i}, \mathrm{DFT}}$ varies from 33 to $44 \mathrm{~kJ} \mathrm{~mol}^{-1}$ and shows a strong correlation with the fitted $\lambda$ values. The systematically lower $\lambda_{\mathrm{i}, \mathrm{DFT}}$ values can be attributed to solvent reorganizations, $\lambda_{\mathrm{o}}$, which are likely significant for the fitted $\lambda$ values but neglected in $\lambda_{\mathrm{i}, \mathrm{DFT}}$. While $\lambda_{\mathrm{i}, \mathrm{DFT}}$ roughly correlates also with calculated $d_{\mathrm{ON}}$ values (Table 4 , Figure S3), it seems that variations in inner sphere reorganization calculated in this fashion are only a minor contribution to the variations in CEPT rates seen experimentally; when the $\lambda_{\mathrm{i}, \mathrm{DFT}}$ values are converted into relative rates (assuming all other parameters are constant, $\Delta \ln k=-\Delta \lambda / 4 R T)$ and these $\ln k_{\text {rel }}$ values are plotted vs experimental $\ln k_{0}$ values, the slope is only 0.12 , indicating that these $\lambda_{\mathrm{i}}$ values would only account for ca. $12 \%$ of the variation in $\ln k_{0}$ (Figure S3).

The values of the electronic coupling, $V_{\mathrm{ET}}$, obtained from the modeling study vary from 1.8 to $6.5 \mathrm{~cm}^{-1}$ (which corresponds to a factor of ca. 10 in rate constant), and follow a similar trend as observed in $\lambda: \mathbf{1}=\mathbf{3}<\mathbf{4}<2$; the trends in $V_{\mathrm{ET}}$ and $\lambda$ have opposite and nearly canceling effects on the rate constants, as discussed above. The decrease of $V_{\mathrm{ET}}$ in $\mathbf{1}$ and 3 relative to 2 and $\mathbf{4}$ could be the result of the donor phenolic HOMO being more delocalized onto the basic heterocycle, away from the Ru moiety. However, the values for $V_{\mathrm{ET}}$ and $\lambda$ which come from this analysis are strongly affected by the variation of $\Delta G^{\circ}$ with temperature $\left(\Delta S^{\circ}\right.$ and $\Delta H^{\circ}$ ). Hupp et al. have reported $\Delta S_{\mathrm{ET}}^{\circ}=115 \mathrm{~J} \mathrm{~mol}^{-1} \mathrm{~K}^{-1}$ for the $\left[\mathrm{Ru}(\mathrm{bpy})_{3}\right]^{3+/ 2+}$ couple in $\mathrm{MeCN}{ }^{68}$ while $\Delta S^{\circ}$ for CEPT oxidations of related phenol-base compounds has been determined to be much smaller both by electrochemistry ${ }^{10-12,39}$ and by chemical equilibration studies. ${ }^{43}$ Given the instability of the phenoxyl radical oxidation product, an experimentally determined $\Delta S^{\circ}$ is problematic, but the variation of the electrochemistry results with temperature for $\mathbf{1 - 4}$ is consistent with literature values for analogues of the $\left[\mathrm{Ru}(\mathrm{bpy})_{3}\right]$ and tyrosinebase units (see the Supporting Information). Various estimates of $\Delta S^{\circ}$ were used in simulation, and all yielded qualitatively similar relative results of $\lambda$ and $V_{\mathrm{ET}}$, and only very small effects on the absolute values of $\kappa_{x}$ and $\Delta E_{a}$, within the series of compounds (see the Supporting Information); therefore, possible reaction entropies do not affect the conclusions of this study.

The numerical modeling scheme also allows for isolation of parameters which are difficult or impossible to achieve experimentally. For example, while changes in proton transfer distance are necessarily correlated to changes in other hydrogen bond parameters, we can use modeling to assess the influence of distance when all other parameters are kept constant. This gives an estimate of the contribution of changes in $r_{0}$ to the differences in CEPT kinetics. Keeping all other parameters equal, such as $\kappa_{x}$, the model predicts $-\partial \ln (k) / \partial r_{0}$ of $15-22$ (Table 4 ) which is on the order of, although smaller than, the $\beta_{\mathrm{H}}$ values predicted from plots of $\ln \left(k_{0}\right)$ vs $d_{\mathrm{ON}}$ or $r_{0}$ in Figure 4. Compound 1 with the very low value of $\kappa_{x}$ (see below) is an outlier here, with $-\partial \ln (k) / \partial r_{0}=7$. When all other parameters are kept constant, this model predicts that the decreased CEPT rate with proton transfer distance will be marked by a higher $E_{\mathrm{a}}$ as a result of the interplay of increased thermal donor-acceptor motions required to compensate for decreased proton tunneling at the equilibrium distance. This trend is indeed observed for the $E_{\mathrm{a}}$ values in Table 2. The prefactor $A$ is expected to decrease with distance, and the ratio $A_{\mathrm{H}} / A_{\mathrm{D}}$ to increase, unless the frequency $(\nu)$ of the 
donor-acceptor effective vibration is low enough that $h \nu \ll k T$; then, the tunneling at high temperature is so efficient that little reduction of $A$ should occur. At intermediate values of $h \nu(h \nu / k T \approx 0.5), A_{\mathrm{H}} / A_{\mathrm{D}}$ can even be $<1 .^{48}$ The data in Table 2 agrees to some extent with this case: $\mathbf{1}, \mathbf{3}$, and $\mathbf{4}$ have essentially equal prefactor values in spite of a large variation in equilibrium tunneling distance, and $\mathbf{1}-\mathbf{3}$ show $A_{\mathrm{H}} / A_{\mathrm{D}}<1$. However, there are two noticeable exceptions: $A$ for 2 , with the largest distance, is higher than that for the other compounds, and for $4, A_{\mathrm{H}} / A_{\mathrm{D}}>1$. The effect of reactant excited states $\left(\Sigma P_{\mu}\right.$ in eq 3$)$ on $A$ and $E_{\mathrm{a}}$ are expected to be small within this model with a Morse potential for the proton and $\nu_{\mathrm{OH}} \sim 3000 \mathrm{~cm}^{-1}$ (see below). Taken in sum, this model predicts that the difference in rates (estimated at $\Delta G^{0}=0 \mathrm{eV}$ ) in $\mathbf{1 - 4}$ is due primarily to changes in the proton transfer distance, with some contribution from the differences in $\kappa_{x}$. However, the values of $\kappa_{x}$ derived from this model, and therefore these conclusions, seem to be problematic, as discussed in the remainder of this section.

The $\Delta E_{\text {a }}$ values obtained from the modeling (Table 4) do not agree within experimental uncertainty with experimental $E_{\mathrm{D}}-$ $E_{\mathrm{H}}$ values (Table 2), although the trend is quite well described. For compounds $1-3, \Delta E_{\mathrm{a}}$ is consistently underestimated by $3.3-4.4 \mathrm{~kJ} \mathrm{~mol}^{-1}$. This is illustrated by the insets of Figure 5, where the model KIEs (dashed line) show a smaller temperature dependence than the experimental data (solid line). For 4 instead, the simulated value is $5.1 \mathrm{~kJ} \mathrm{~mol}^{-1}$, while the experimental KIE is essentially independent of temperature, in contrast with bimolecular studies on the analogue $4^{\prime}$, mentioned above. The low modeled values of $\Delta E_{\mathrm{a}}$ for $\mathbf{1 - 3}$ are one indication that the magnitude of $\kappa_{x}$ values obtained from the modeling are underestimated; this results in an overestimation of the tunneling distances that can be sampled at a given temperature, which favors tunneling for the deutero analogue relative to the proteo and therefore results in a decreased $\Delta E_{\mathrm{a}}$.

Values for $\kappa_{x}$ from the modeling studies range from 3.9 to 21.2 $\mathrm{J} \mathrm{m}^{-2}$ (Table 4) and follow the trend $\mathbf{1} \ll \mathbf{3}<\mathbf{2}<\mathbf{4}$. This effective value of $\kappa_{x}$ derived from the numerical modeling of the experimental kinetic data is most likely a sum of contributions from several normal modes projected on the donor-acceptor vector. For $1, \kappa_{x}$ is very low, a result of the low KIE and relatively long $r_{0}$. These values of $\kappa_{x}$ give an indication of the importance of proton donor-acceptor motions on CEPT reactivity. However, it appears that the absolute values of $\kappa_{x}$ are severely underestimated here. The values of $\kappa_{x}$ derived from modeling result in the prediction that CEP occurs primarily at configurations where $r$ is compressed by ca. $0.41-0.56 \AA$ (compound $\mathbf{1}$ is again an outlier with $0.75 \AA$ ) ; see the Supporting Information. However, DFT calculations predict that distortions of that magnitude would result in energetic barriers of ca. $40-100 \mathrm{~kJ} \mathrm{~mol}^{-1}$ for 2-4, much higher than experimental $E_{\mathrm{a}}$ values; this is an indication that the fitted $\kappa_{x}$ values are unreasonably low. Some possible reasons for this discrepancy will be discussed below; however, we will generally focus on the trends within the series because of the various assumptions in the model, e.g., constant hydrogen bond potentials that are independent of proton donor-acceptor motions.

The values of $\kappa_{x}$ are predicted to be higher for the nonconjugated species, as also noted for the previously studied phenol-carboxylates. ${ }^{18}$ However, in previous studies of $3^{\prime}$ and $4^{\prime}$ and related compounds, it has been noted that $\kappa_{x}$, as determined from potential energy scans of $d_{\mathrm{ON}}$, followed a trend of higher values of $\kappa_{x}$ being observed with shorter $d_{\mathrm{ON}} \cdot{ }^{69,43}$ Such potential energy scans should report on the energy-weighted contributions of proton donor-acceptor motions through the various contributing normal modes when the motions of all nuclei are adiabatic. ${ }^{23}$ When similar scans of the $\mathrm{O} \cdots \mathrm{N}$ coordinate were performed for 1-4, it was found that the nonconjugated compounds, with longer $d_{\mathrm{ON}}$ and higher KIE, have lower values of $\kappa_{x}$. Note that calculations performed using a range of basis sets and levels of theory (e.g., in the presence of additional diffuse or polarization functions in the basis set, B3LYP and BPEBPE density functionals, with and without explicit solvent molecule or PCM solvent model, Hartree-Fock-based MP2 method, and semiempirical PM3) all predict the same trend for 3 and 4; see the Supporting Information. Further, there is a strong linear correlation between the DFT-derived values of $\kappa_{x}$ and $d_{\mathrm{ON}}$ among the oxidized and reduced forms of 1-4 (Figure 7).

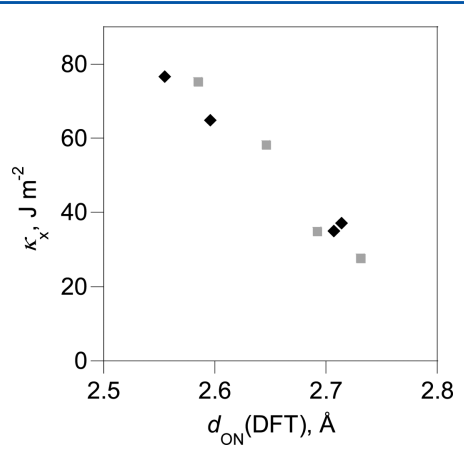

Figure 7. Variation of $\kappa_{x}$, the force constant for compression of $d_{\mathrm{ON}}$, obtained from DFT pontential energy surface scans of $d_{\mathrm{ON}}$ about the equilibrium value, $d_{\mathrm{ON}}(\mathrm{DFT})$, for each modified tyrosine, $1-4$, in the reduced (black diamonds) and oxidized (gray squares) forms.

An inverse relation between $\kappa_{x}$ and $r_{0}$ has also been suggested in some pressure-dependent kinetic studies of enzymatic systems. $31,70,71$

In order to probe whether the $\mathrm{O} \cdots \mathrm{N}$ potential energy scans fail to include key motions that are important in the CEPT reactivity in 1-4, molecular dynamics simulations were performed using the GAMESS software package. ${ }^{72,73}$ These simulations used the semiempirical PM3 level of theory. While this is a relatively low level of theory which is not optimized for hydrogen bonding interactions, $\mathrm{PM} 3$ potential energy scans yielded the same trends in $\kappa_{x}$ as higher level DFT and MP2 calculations, and should therefore suffice to validate the methodology. Further, this method was used to provide qualitative support of trends in donor-acceptor motions in a related study. ${ }^{18}$ Following that study, 40 ps constant energy simulations were run following 3 ps of thermal equilibration. This equilibration was confirmed to be sufficient, as analysis of the first and second 20 ps of the simulations independently gave identical results. The simulations reported here were performed at $100 \mathrm{~K}$. The distribution of $d_{\mathrm{ON}}$ throughout the MD simulations is plotted in Figure 8. When fitting the compressed $d_{\mathrm{ON}}$ values to a harmonic oscillator, the resulting force constants follow the trend $2 \sim 4<3<1$ (Table S6), indicating general agreement between MD simulations and PES scans. Spectral densities of proton donoracceptor motions have been calculated from the MD simulations following ref 18 and can be found in the Supporting Information. These show that donor-acceptor compression in the nonconjugated analogues requires lower energy, compared to the conjugated compounds. In conclusion, the computational results contradict the trend in $\kappa_{x}$ suggested by the kinetic modeling.

To summarize the results of kinetic modeling, the dependence of CEPT rates on proton transfer distance is qualitatively predicted 

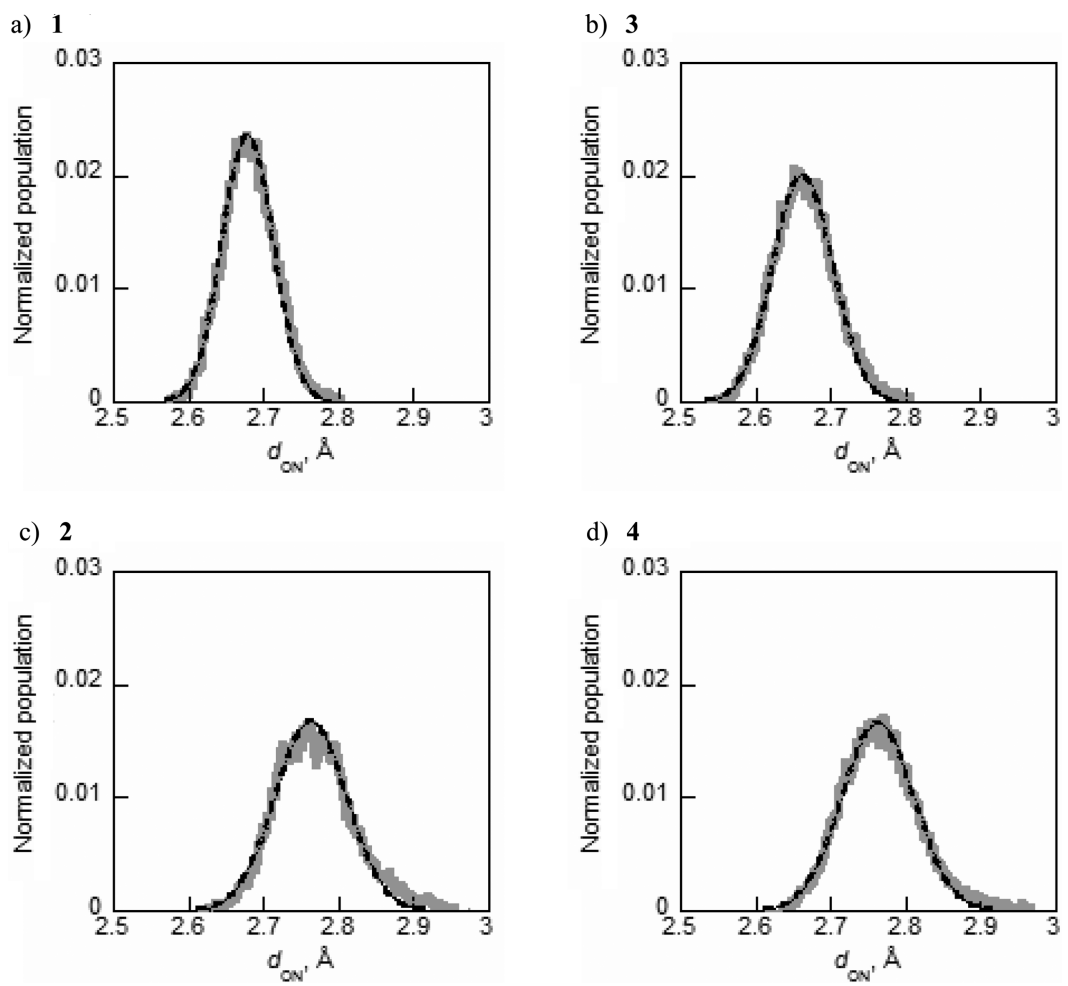

Figure 8. Distribution of $d_{\mathrm{ON}}$ in molecular dynamics simulations (gray line) for compounds 1-4. The black dashed line is a fit to a harmonic oscillator (fitted only to points where $d_{\mathrm{ON}}$ was compressed below the optimized value).

by the relatively simple Kuznetsov-Ulstrup model. However, the model appears to have trouble describing the experimental KIE values for the present series of compounds, because the trends for the donor-acceptor vibrations, $\kappa_{x}$, appear to be too low and, more importantly, the trend in $\kappa_{x}$ is opposite to that predicted independently by various computational methodologies. This difficulty, together with the fact that the modeled changes in the other two fitted parameters, $\lambda$ and $V_{\mathrm{ET}}$, essentially cancel each other out, limits the amount of further insights into the trends in reactivity displayed in Figure 4. Several possible origins of these problems are described in the next section.

Possible Origins of Shortcomings of the KuznetsovUlstrup Model in the Present Case. The computation of $r_{0}$ is a key input to the modeling and has a strong dependence on the angle of the hydrogen bond in the oxidized, radical product. It is possible that DFT does a poor job of predicting the hydrogen bond geometries of these radical cation species. Alternatively, the differences in equilibrium hydrogen bond angle in the product state may in fact not be an important parameter through the course of donor-acceptor motions that distort the hydrogen bond to the configurations where the tunneling occurs. In order to probe whether these geometrical effects on $r_{0}$ are the cause of significant artifacts, the analysis was repeated using a proton tunneling distance which scales with $d_{\mathrm{ON}}$. The assumption of a linear hydrogen bond with typical $\mathrm{OH}$ and $\mathrm{NH}$ bond lengths of 0.97 and $1.03 \AA$, respectively, ${ }^{74}$ leads to a crude estimation of an equilibrium proton tunneling distance of $d_{\mathrm{ON}}-2.00 \AA$, which ranges from 0.549 to $0.707 \AA$. While this is obviously a poor description of the equilibrated proton transfer distance, it might be a reasonable starting point for an effective tunneling distance in this model, if the hydrogen bond becomes significantly more linear via donor-acceptor vibrations. The details of this treatment can be found in the Supporting Information. In sum, it leads to an increase in the magnitude of $\kappa_{x}$ across the series (although in 1 the increase is only from 3.9 to $6.4 \mathrm{~J} \mathrm{~m}^{-2}$ ); however, the overall trends in $\kappa_{x}$ across the series remain unchanged. Agreement with experimental $\Delta E_{\mathrm{a}}$ values also does not improve.

Variations in $k_{\mathrm{H}}$ or KIE with $\Delta G^{\circ}$ could also be a source of error in the fitted parameters. The driving force for CEPT in 1-4 varies, and the systems with the more downhill $\Delta G^{\circ}$ have slower rates and higher KIE. The model used here predicts the same variation of rates with driving force as was used to calculate $k_{0}$, i.e., $\alpha=1 / 2$; however, variations in $\alpha$ have been observed in related phenol oxidations from ca. $0.6^{11}$ to 0.3 . $^{43}$ The model also predicts that KIE should be relatively independent from $\Delta G^{\circ}$ for these compounds. There are some indications in the literature that KIE for PCET reactions may have an unusual dependence on driving force. Semiclassical and more advanced models predict that KIE should reach a maximum near $\Delta G^{\circ}=0$ for hydrogen transfer reactions, ${ }^{58,59,75,76}$ as has been observed in a number of systems. ${ }^{77,78}$ However, increases in KIE with more downhill reactions have been observed elsewhere. ${ }^{43,79,80}$ Because the range in KIE here is fairly small, subtle differences in, e.g., vibrational excited states not accounted for in this treatment could yield spurious fitted parameters.

Another possible source of error which might lead to the discrepancy in the trends of $\kappa_{x}$ as well as the apparent underestimation of the absolute values in this series of compounds is the variation of the hydrogen bond potentials with changes in $d_{\mathrm{ON}}$. This model assumes that product and reactant hydrogen bond potentials remain constant as the proton donor-acceptor distance fluctuates. However, the strength of the hydrogen bonding interaction, and therefore the hydrogen bond potential, should be strongly affected by changes in $d_{\mathrm{ON}}$; specifically, decreases in $d_{\mathrm{ON}}$ should result in decreases in both $\nu_{\mathrm{XH}}$ and $D_{\mathrm{e}}$. These changes should result in higher fitted values of $\kappa_{x}$, as less distortion is required to achieve the necessary overlap. This effect 
a) 1 (red)

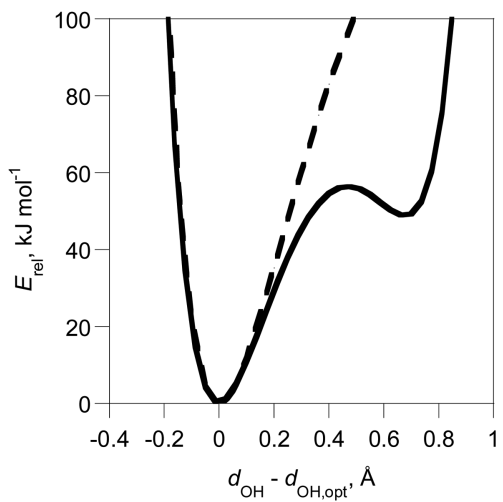

c) 2 (red)

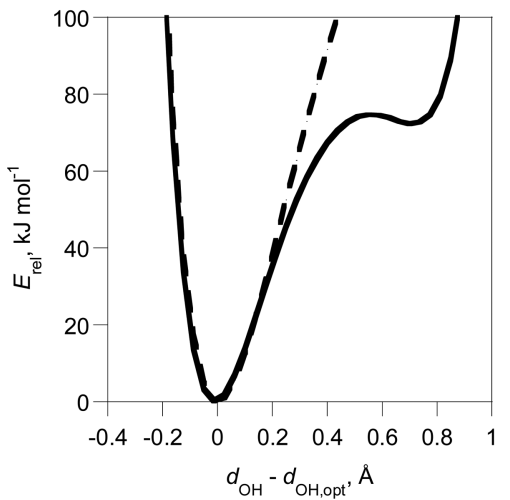

b) 1 (ox)

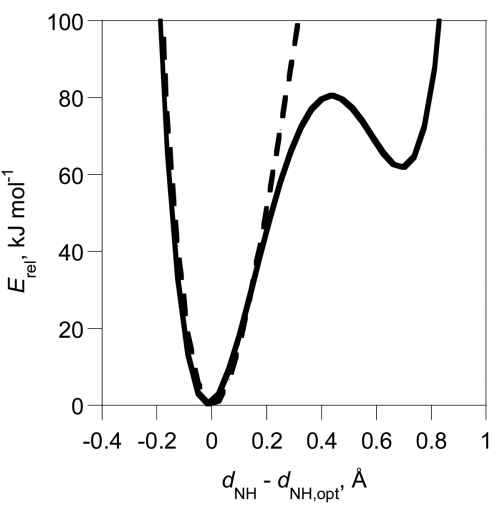

d) 2 (ox)

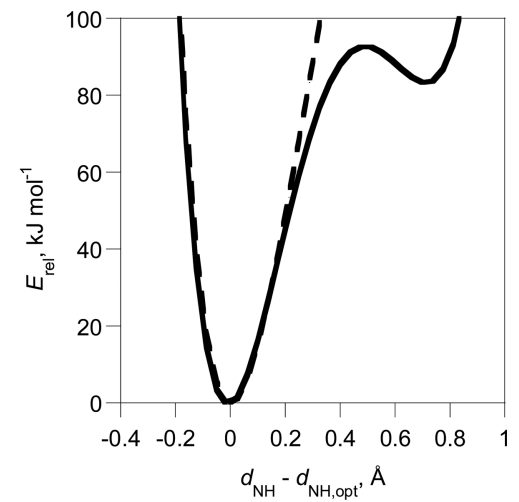

Figure 9. DFT calculated hydrogen bond potentials for reduced (left) and oxidized (right) forms of $\mathbf{1}$ and $\mathbf{2}$. Dashed lines are Morse potentials derived from rigid scans of $d_{\mathrm{XH}}$, and solid lines are semirelaxed scans where the hydrogen bond angle is also allowed to vary, with all other atoms fixed.

was probed computationally, and the variation of the hydrogen bond potential with $d_{\mathrm{ON}}$ was included in the numerical modeling. The details of this treatment can be found in the Supporting Information; however, to summarize, the magnitude of $\Delta E_{\mathrm{a}}$ and $\kappa_{x}$ values are consistently increased by including this effect, yielding results more in line with experiment, but the trend in the $\kappa_{x}$ values is not changed.

Finally, another possible source of error that could contribute to the discrepancy in the trends in $\kappa_{x}$ lies in the assumption of a Morse potential for the hydrogen bond. The plots in Figure 9 compare the Morse potential derived from rigid scans of $d_{\mathrm{XH}}$ at ca. $\pm 0.1 \AA$ around the optimized value with the partially relaxed proton transfer potential energy surface (PT-PES) from points calculated across the entire H-bond distance optimizing the proton position within the optimized (and fixed) heavy-atom framework. While the Morse potential and the PT-PES agree quite well near the minimum, there are significant deviations at proton positions far from the equilibrium position leading to double well potentials. These highly anharmonic, non-Morse and/or double well potentials will lead to energetically accessible vibrationally excited states that have significant density far away from the equilibrium position. This is significant, especially for the product states, because it is in this region where the FranckCondon overlap of reactant and product proton wave functions will be most efficient. In a number of previous computational studies of CEPT where proton potential energy surfaces are treated explicitly, it has been found that pathways from the ground state to vibrationally excited states were significant contributors to CEPT reactivity, particularly in the deuterium case and at longer PT transfer distances. ${ }^{23,24,63,69,81-83}$ In contrast, while the KuznetsovUlstrup model used here formally includes all accessible excited states for both the reactant and product, it predicts that these excited states are very minor contributors to CEPT reactivity (<ca. 10\% in all systems). It appears that the use of Morse hydrogen bond potentials leads to an underestimation of excited state contributions, and hence the problems fitting the experimental trends in KIE. For example, in Figure 9, the $\mathrm{O}-\mathrm{H}$ bound form of oxidized 1-(P) is ca. $20 \mathrm{~kJ} \mathrm{~mol}^{-1}$ lower than that of 2 -(P). This would favor pathways to vibrationally excited states. Further, each of the treatments pictured in Figure 9 reduces the transferring proton to a one-dimensional $\mathrm{X}-\mathrm{H}$ stretching surface (although the partially relaxed PT-PES surfaces include some bending motions). Recently, Liu et al. have reported a theoretical study of CEPT reactions of $3^{\prime}$, using 2-D hydrogen bond potentials that include $\mathrm{C}-\mathrm{X}-\mathrm{H}$ bending within the plane of conjugated rings. ${ }^{83}$ They found that low-lying vibrational excited states corresponding to $\mathrm{O}-\mathrm{H}$ bending are involved in CEPT reactivity; however, their effect on the rates and KIE were not large. Also, bending motions are likely less important in $\mathbf{2}$ and $\mathbf{4}$ which have more linear hydrogen bonds. In sum, the assumption of a Morse hydrogen bond potential likely leads to an underestimation of the influence of vibrational excited states, particularly for the conjugated $\mathbf{1}$ and 3 . This could lead to increased $k_{0}$ and decreased KIE for $\mathbf{1}$ vs $\mathbf{2}$. Most importantly, it may also be an explanation for the discrepancies in the trend of $\kappa_{x}$, as well as the absolute values, which is fit to the experimental KIE.

In summary, there are a number of uncertainties and assumptions in the model as implemented. These include the correct determination of the equilibrium proton transfer distance, as well as $\mathrm{X}-\mathrm{H}$ stretch frequencies and hydrogen bond potentials; also, the reduction of the PT coordinate to a single dimension. A number of them will be explored in greater detail, in particular the assumption of the Morse potentials. 


\section{CONCLUSIONS}

In this series of related ruthenium-linked tyrosine-base compounds 1-4, CEPT rates decrease with more favorable $\Delta G^{\circ}$, which is contrary to what is expected for moderate driving forces. However, when the rates are extrapolated to $\Delta G^{\circ}=0$, it becomes evident that other factors are at play. These driving force corrected rates, $k_{0}$, generally correlate with proton transfer distance. First, however, it should be noted that, even in these small, relatively well-defined intramolecularly hydrogen bonded systems, precise determination of the relevant proton transfer distance via crystallographic or DFT determined $d_{\mathrm{ON}}$ values, or estimated equilibrium proton tunneling distance, $r_{0}$, can be problematic. Second, while the magnitude of the dependence of $k_{0}$ upon $d_{\mathrm{ON}}(\beta)$ is on the order of that predicted by simple tunneling models, this treatment (eq 11) assumes that all other parameters remain constant, which is likely not the case. In this series of compounds, $d_{\mathrm{ON}}$ is also correlated with other parameters reporting on variations of the hydrogen bond, in large part due to the differences arising from the rigid $\pi$ system in the conjugated compounds $\mathbf{1}$ and 3 vs the more flexible sevenmembered ring in $\mathbf{2}$ and $\mathbf{4}$.

The experimental data indicates that, in this series, the KIE increases with increased proton transfer distance, and while this is what is expected when only the distance is varied, this result is not general. The opposite trend-a decrease in KIE with increased PT distance-has been observed in other systems ${ }^{43,69}$ and ascribed to changes in the force constant for proton donoracceptor motions and anharmonicity of the hydrogen bond potential that varies with proton donor-acceptor distance. ${ }^{43,76}$ From the temperature dependence of the kinetics for $\mathbf{1 - 4}$, it is evident that the variation in reactivity is primarily a consequence of temperature-dependent energetic terms (Arrhenius $E_{\mathrm{a}}$ ). This highlights the interplay of energetic donor-acceptor motions, thermal population of proton vibrational excited states, and the otherwise temperature independent proton tunneling probabilites. Proton tunneling is indicated by the temperature dependence of KIE; however, closer examination of the data, specifically fitting the kinetic data to the tunneling model of Kuznetsov and Ulstrup (with inclusion of proton donor-acceptor motions), reveals that this model appears inadequate to capture the factors which are influencing reactivity. This model predicts that the increased KIE in compounds with longer $d_{\mathrm{ON}}$ is a result of higher effective force constant for proton donor-acceptor motions $\left(\kappa_{x}\right)$. However, the opposite trend-that $\kappa_{x}$ decreases with $d_{\mathrm{ON}}$-is found when $\kappa_{x}$ is directly probed computationally via either potential energy scans or MD simulations. This discrepancy suggests that the model is missing a key feature in the reactivity of these compounds. We tentatively suggest that a Morse potential treatment for the proton potential of strongly hydrogen-bonded systems is inadequate in many cases and could be the cause of the apparent qualitative discrepancies obtained here. Modeling with more accurate proton potentials is more cumbersome but may be required to properly analyze differences in reactivity between related compounds; see, e.g., refs 23, 24, 63, 69, and 81-83. This finding for the small synthetic compounds $\mathbf{1}-\mathbf{4}$ should be considered also for larger and more complex systems, such as proteins.

\section{EXPERIMENTAL SECTION}

Compounds 1-4 were available from a previous study. ${ }^{19}$ All samples were prepared in spectroscopic grade acetonitrile $\left(0.75 \% \mathrm{H}_{2} \mathrm{O}\right.$ or $\left.\mathrm{D}_{2} \mathrm{O}\right)$ with $20-50 \mathrm{mM}$ methylviologen $\left(\mathrm{MV}\left(\mathrm{PF}_{6}\right)_{2}\right){ }^{19}$ Kinetic experiments were performed with an Applied Photophysics LKS80 spectrometer and a nanosecond
YAG-laser/OPO combination (Quantel Brilliant B/OPOTEK), as described before. ${ }^{17,18,37,56}$ Briefly, the sample was excited with $\sim 10 \mathrm{~ns}, 10 \mathrm{~mJ}$ laser flashes at $460-480 \mathrm{~nm}$. The transient absorption kinetics was monitored with a pulsed Xe lamp, a monochromator, and PMT detector, feeding a digital oscilloscope. The data was converted to transient absorption and fitted using the LKS80 software, or fitted by Origin software. Each kinetic trace is an average of 4-16 shots. The instrumental response function has a fwhm $<15 \mathrm{~ns}$. The temperature was controlled by circulating water from a heater/chiller in the sample holder, and monitored by a standard thermometer.

\section{ASSOCIATED CONTENT}

\section{S Supporting Information}

The Supporting Information is available free of charge on the ACS Publications website at DOI: 10.1021/acs.jpcb.6b05885.

Additional kinetic and computation details and data (PDF)

\section{AUTHOR INFORMATION}

Corresponding Author

*E-mail: leif.hammarstrom@kemi.uu.se. Phone: +46-18-471 3648.

\section{Present Addresses}

${ }^{\dagger}$ T.F.M.: Department of Chemistry, Yale University, 225 Prospect Street, P.O. Box 208107, New Haven, CT 065208107, USA.

${ }^{\ddagger}$ M.-T.Z.: Department of Chemistry, Tsinghua University, Beijing 100084, China.

${ }^{\S}$ M.-P.S.: Dipartimento di Scienze Chimiche, Università di Messina, Via F. Stagno d'Alcontres 31, Messina, Italy.

"L.O.J.: School of Chemical Engineering and Analytical Science, The University of Manchester Manchester, M13 9PL, U.K.

Notes

The authors declare no competing financial interest.

\section{ACKNOWLEDGMENTS}

We thank Daniel Spångberg for assistance with molecular dynamics simulations and Reuben Jane and Andeas Orthaber for additional assistance with the crystallographic structure of $\mathbf{2}^{\prime}$. This work was supported by the Swedish Research Council, the Swedish Energy Agency, and the Knut and Alice Wallenberg Foundation.

\section{REFERENCES}

(1) Cukier, R. I.; Nocera, D. G. Proton-coupled electron transfer. Annu. Rev. Phys. Chem. 1998, 49, 337-369.

(2) Weinberg, D. R.; Gagliardi, C. J.; Hull, J. F.; Murphy, C. F.; Kent, C. A.; Westlake, B. C.; Paul, A.; Ess, D. H.; McCafferty, G.; Meyer, T. J. Proton-Coupled Electron Transfer. Chem. Rev. 2012, 112, 4016-4093.

(3) Reece, S. Y.; Nocera, D. G. Proton-Coupled Electron Transfer in Biology: Results from Synergistic Studies in Natural and Model Systems. Annu. Rev. Biochem. 2009, 78, 673-699.

(4) Proton-Coupled Electron Transfer. Thematic Issue. Chem. Rev. 2010, 110, 6937.

(5) Hammes-Schiffer, S. Proton-Coupled Electron Transfer: Moving Together and Charging Forward. J. Am. Chem. Soc. 2015, 137, 88608871.

(6) Hydrogen-Transfer Reactions; Hynes, J. T., Klinman, J. P., Limbach, H. H., Schowen, R. L., Eds.; Wiley: Weinheim, Germany, 2007.

(7) Cox, N.; Retegan, M.; Neese, F.; Pantazis, D. A.; Boussac, A.; Lubitz, W. Electronic structure of the oxygenevolving complex in 
photosystem II prior to O-O bond formation. Science 2014, 345, 804808.

(8) Klauss, A.; Haumann, M.; Dau, H. Alternating electron and proton transfer steps in photosynthetic water oxidation. Proc. Natl. Acad. Sci. U. S. A. 2012, 109, 16035-16040.

(9) Li, X.; Siegbahn, P. E. M.; Ryde, U. Simulation of the isotropic EXAFS spectra for the S-2 and S-3 structures of the oxygen evolving complex in photosystem II. Proc. Natl. Acad. Sci. U. S. A. 2015, 112, 3979-3984.

(10) Costentin, C.; Robert, M.; Savéant, J.-M. Carboxylates as protonaccepting groups in concerted proton-electron transfers. Electrochemistry of the 2,5-dicarboxylate 1,4-hydrobenzoquinone/2,5-dicarboxy 1,4-benzoquinone couple. J. Am. Chem. Soc. 2006, 128, 87268727.

(11) Costentin, C.; Robert, M.; Savéant, J.-M. Adiabatic and nonadiabatic concerted proton-electron transfers. Temperature effects in the oxidation of intramolecularly hydrogen-bonded phenols. J. Am. Chem. Soc. 2007, 129, 9953-9963.

(12) Correction to ref 11: Costentin, C.; Robert, M.; Savéant, J.-M. Adiabatic and Non-adiabatic Concerted Proton-Electron Transfers. Temperature Effects in the Oxidation of Intramolecularly HydrogenBonded Phenols (vol 129, pg 9953, 2007). J. Am. Chem. Soc. 2010, 132, 2845-2845.

(13) Fecenko, C. J.; Thorp, H. H.; Meyer, T. J. The role of free energy change in coupled electron-proton transfer. J. Am. Chem. Soc. 2007, 129, 15098-15099.

(14) Pizano, A. A.; Yang, J. L.; Nocera, D. G. Photochemical tyrosine oxidation with a hydrogen-bonded proton acceptor by bidirectional proton-coupled electron transfer. Chem. Sci. 2012, 3, 2457-2461.

(15) Kuss-Petermann, M.; Wenger, O. S. Influence of Donor-Acceptor Distance Variation on Photoinduced Electron and Proton Transfer in Rhenium(I)-Phenol Dyads. J. Phys. Chem. A 2013, 117, 5726-5733.

(16) Herzog, W.; Bronner, C.; Löffler, S.; He, B.; Kratzert, D.; Stalke, D.; Hauser, A.; Wenger, O. S. Electron Transfer between HydrogenBonded Pyridylphenols and a Photoexcited Rhenium(I) Complex. ChemPhysChem 2013, 14, 1168-1176.

(17) Irebo, T.; Johansson, O.; Hammarström, L. The Rate Ladder of Proton-Coupled Tyrosine Oxidation in Water: A Systematic Dependence on Hydrogen Bonds and Protonation State. J. Am. Chem. Soc. 2008, 130, 9194-9195.

(18) Johannissen, L. O.; Irebo, T.; Sjödin, M.; Johansson, O.; Hammarström, L. The Kinetic Effect of Internal Hydrogen Bonds on Proton-Coupled Electron Transfer from Phenols: A Theoretical Analysis with Modeling of Experimental Data. J. Phys. Chem. B 2009, 113, 16214-16225.

(19) Zhang, M.-T.; Irebo, T.; Johansson, O.; Hammarström, L. Proton-Coupled Electron Transfer from Tyrosine: A Strong Rate Dependence on Intramolecular Proton Transfer Distance. J. Am. Chem. Soc. 2011, 133, 13224-13227.

(20) Lachaud, F.; Quaranta, A.; Pellegrin, Y.; Dorlet, P.; Charlot, M.-F.; Un, S.; Leible, W.; Aukauloo, A. A biomimetic model of the electron transfer between P-680 and the TyrZ-His 190 pair of PSII. Angew. Chem., Int. Ed. 2005, 44, 1536-1540.

(21) Megiatto, J. D., Jr; Méndez-Hernández, D. D.; Tejeda-Ferrari, M. E.; Teillout, A.-L.; Llansola-Portolés, M. J.; Kodis, G.; Poluektov, O. G.; Rajh, T.; Mujica, V.; Groy, T. L.; Gust, D.; Moore, T. A.; Moore, A. L. A bioinspired redox relay that mimics radical interactions of the Tyr-His pairs of photosystem II. Nat. Chem. 2014, 6, 423-428.

(22) Benisvy, L.; Bittl, R.; Bothe, E.; Garner, C. D.; McMaster, J.; Ross, S.; Teutloff, C.; Neese, F. Phenoxyl radicals hydrogen-bonded to imidazolium: Analogues of tyrosyl D of photosystem II: High-field EPR and DFT studies. Angew. Chem., Int. Ed. 2005, 44, 5314-5317.

(23) Auer, B.; Fernandez, L. E.; Hammes-Schiffer, S. Theoretical Analysis of Proton Relays in Electrochemical Proton-Coupled Electron Transfer. J. Am. Chem. Soc. 2011, 133, 8282-8292.

(24) Skone, J. H.; Soudackov, A. V.; Hammes-Schiffer, S. Calculation of vibronic couplings for phenoxyl/phenol and benzyl/toluene selfexchange reactions: Implications for proton-coupled electron transfer mechanisms. J. Am. Chem. Soc. 2006, 128, 16655-16663.
(25) Pu, J.; Gao, J.; Truhlar, D. G. Mechanisms and free energies of enzymatic reactions. Chem. Rev. 2006, 106, 3140-3169.

(26) Klinman, J. P. The role of tunneling in enzyme catalysis of C-H activation. Biochim. Biophys. Acta, Bioenerg. 2006, 1757, 981-987.

(27) Hatcher, E.; Soudackov, A. V.; Hammes-Schiffer, S. Protoncoupled electron transfer in soybean lipoxygenase. J. Am. Chem. Soc. 2004, 126, 5763-5775.

(28) Hatcher, E.; Soudackov, A. V.; Hammes-Schiffer, S. Protoncoupled electron transfer in soybean lipoxygenase: Dynamical behavior and temperature dependence of kinetic isotope effects. J. Am. Chem. Soc. 2007, 129, 187-196.

(29) Masgrau, L.; Roujeinikova, A.; Johannissen, L. O.; Hothi, P.; Basran, J.; Ranaghan, K. E.; Mulholland, A. .; Sutcliffe, M. J.; Scrutton, N. S.; Leys, D. Atomic description of an enzyme reaction dominated by proton tunneling. Science 2006, 312, 237.

(30) Johannissen, L. O.; Hay, S.; Scrutton, N. S.; Sutcliffe, M. J. Proton tunneling in aromatic amine dehydrogenase is driven by a short-range sub-picosecond promoting vibration: Consistency of simulation and theory with experiment. J. Phys. Chem. B 2007, 111, 2631-2638.

(31) Hay, S.; Sutcliffe, M. J.; Scrutton, N. S. Promoting motions in enzyme catalysis probed by pressure studies of kinetic isotope effects. Proc. Natl. Acad. Sci. U. S. A. 2007, 104, 507-512.

(32) Pudney, C. R.; Johannissen, L. O.; Sutcliffe, M. J.; Hay, S.; Scrutton, N. S. Direct Analysis of Donor Acceptor Distance and Relationship to Isotope Effects and the Force Constant for Barrier Compression in Enzymatic H-Tunneling Reactions. J. Am. Chem. Soc. 2010, 132, 11329-11335.

(33) Peters, B. Transition-State Theory, Dynamics, and Narrow Time Scale Separation in the Rate-Promoting Vibrations Model of Enzyme Catalysis. J. Chem. Theory Comput. 2010, 6, 1447-1454.

(34) Benkovic, S. J.; Hammes-Schiffer, S. A perspective on enzyme catalysis. Science 2003, 301, 1196-1202.

(35) Hammes-Schiffer, S.; Stuchebrukhov, A. A. Theory of Coupled Electron and Proton Transfer Reactions. Chem. Rev. 2010, 110, 69396960.

(36) Reece, S. Y.; Nocera, D. G. Direct tyrosine oxidation using the MLCT excited states of rhenium polypyridyl complexes. J. Am. Chem. Soc. 2005, 127, 9448-9458.

(37) Irebo, T.; Reece, S. Y.; Sjodin, M.; Nocera, D. G.; Hammarstrom, L. Proton-coupled electron transfer of tyrosine oxidation: Buffer dependence and parallel mechanisms. J. Am. Chem. Soc. 2007, 129, 15462 .

(38) Ishikita, H.; Soudackov, A. V.; Hammes-Schiffer, S. Buffer-assisted proton-coupled electron transfer in a model rhenium-tyrosine complex. J. Am. Chem. Soc. 2007, 129, 11146-11152.

(39) Rhile, I. J.; Markle, T. F.; Nagao, H.; DiPasquale, A. G.; Lam, O. P.; Lockwood, M. A.; Rotter, K.; Mayer, J. M. J. Am. Chem. Soc. 2006, $128,6075-6088$.

(40) Markle, T. F.; Mayer, J. M. Concerted proton-electron transfer in pyridylphenols: The importance of the hydrogen bond. Angew. Chem., Int. Ed. 2008, 47, 738-740.

(41) Markle, T. F.; Rhile, I. J.; Mayer, J. M.; DiPasquale, A. G. Probing concerted proton-electron transfer in phenol-imidazoles. Proc. Natl. Acad. Sci. U. S. A. 2008, 105, 8185-8190.

(42) Markle, T. F.; Tronic, T. A.; DiPasquale, A. G.; Kaminsky, W.; Mayer, J. M. Effect of Basic Site Substituents on Concerted ProtonElectron Transfer in Hydrogen-Bonded Pyridyl-Phenols. J. Phys. Chem. A 2012, 116, 12249-12259.

(43) Markle, T. F.; Rhile, I. J.; Mayer, J. M. Kinetic Effects of Increased Proton Transfer Distance on Proton-Coupled Oxidations of PhenolAmines. J. Am. Chem. Soc. 2011, 133, 17341-17352.

(44) Marcus, R. A.; Sutin, N. Electron Transfers in Chemistry and Biology. Biochim. Biophys. Acta, Rev. Bioenerg. 1985, 811, 265-322.

(45) Carra, C.; Iordanova, N.; Hammes-Schiffer, S. Proton-coupled electron transfer in a model for tyrosine oxidation in photosystem II. J. Am. Chem. Soc. 2003, 125, 10429-10436.

(46) Soudackov, A.; Hatcher, E.; Hammes-Schiffer, S. Quantum and dynamical effects of proton donor-acceptor vibrational motion in 
nonadiabatic proton-coupled electron transfer reactions. J. Chem. Phys. 2005, 122, 014505 .

(47) Kuznetsov, A. M.; Ulstrup, J. Proton and hydrogen atom tunnelling in hydrolytic and redox enzyme catalysis. Can. J. Chem. 1999, 77, 1085-1096.

(48) Knapp, M. J.; Rickert, K.; Klinman, J. P. Temperature-dependent isotope effects in soybean lipoxygenase-1: Correlating hydrogen tunneling with protein dynamics. J. Am. Chem. Soc. 2002, 124, 38653874.

(49) Meyer, M. P.; Klinman, J. P. Modeling temperature dependent kinetic isotope effects for hydrogen transfer in a series of soybean lipoxygenase mutants: The effect of anharmonicity upon transfer distance. Chem. Phys. 2005, 319, 283-296.

(50) Benisvy, L.; Bill, E.; Blake, A. J.; Collison, D.; Davies, E. S.; Garner, C. D.; McArdle, G.; McInnes, E. J. L.; McMaster, J.; Ross, S. H. K.; Wilson, C. Phenoxyl radicals: H-bonded and coordinated to $\mathrm{Cu}(\mathrm{II})$ and Zn(II). Dalton Trans 2006, 258-267.

(51) Orio, M.; Jarjayes, O.; Baptiste, B.; Philouze, C.; Duboc, C.; Mathias, J.-L.; Benisvy, L.; Thomas, F. Geometric and Electronic Structures of Phenoxyl Radicals Hydrogen Bonded to Neutral and Cationic Partners. Chem. - Eur. J. 2012, 18, 5416-5429.

(52) Frisch, M. J.; Trucks, G. W.; Schlegel, H. B.; Scuseria, G. E.; Robb, M. A.; Cheeseman, J. R.; Scalmani, G.; Barone, V.; Mennucci, B.; Petersson, G. A.; et al. Gaussian 09, revision C.01; Gaussian, Inc.: Wallingford, CT, 2010. See the Supporting Information for the complete reference.

(53) Gilli, P.; Bertolasi, V.; Ferretti, V.; Gilli, G. Evidence for intramolecular $\mathrm{N}-\mathrm{H} \cdots \mathrm{O}$ resonance-assisted hydrogen bonding in betaenaminones and related heterodienes. A combined crystal-structural, IR and NMR spectroscopic, and quantum-mechanical investigation. J. Am. Chem. Soc. 2000, 122, 10405-10417.

(54) Gilli, P.; Pretto, L.; Bertolasi, V.; Gilli, G. Predicting HydrogenBond Strengths from Acid-Base Molecular Properties. The pK(a) Slide Rule: Toward the Solution of a Long-Lasting Problem. Acc. Chem. Res. 2009, 42, 33-44.

(55) Sanz, P.; Mó, O.; Yáñez, M.; Elguero, J. Resonance-assisted hydrogen bonds: A critical examination. Structure and stability of the enols of beta-diketones and beta-enaminones. J. Phys. Chem. A 2007, $111,3585-3591$.

(56) Magnuson, A.; Berglund, H.; Korall, P.; Hammarström, L.; Åkermark, B.; Styring, S.; Sun, L. Mimicking electron transfer reactions in photosystem II: Synthesis and photochemical characterization of a ruthenium(II) tris(bipyridyl) complex with a covalently linked tyrosine. J. Am. Chem. Soc. 1997, 119, 10720-10725.

(57) Given the magnitude of $\Delta G^{\circ}$ for these reactions, it is not certain that they fall in the regime where $\alpha=1 / 2$. However, the differences in intrinsic reactivity between compounds 1,3 , and 4 is in good agreement with previously reported bimolecular stopped-flow kinetics on $\mathbf{1}^{\prime}, \mathbf{3}^{\prime}$, and $\mathbf{4}^{\prime}$ (refs 39 and 40 ).

(58) Kiefer, P. M.; Hynes, J. T. Kinetic isotope effects for nonadiabatic proton transfer reactions in a polar environment. 1. Interpretation of tunneling kinetic isotopic effects. J. Phys. Chem. A 2004, 108, 1179311808 .

(59) Kiefer, P. M.; Hynes, J. T. Kinetic isotope effects for nonadiabatic proton transfer reactions in a polar environment. 2. Comparison with an electronically diabatic description. J. Phys. Chem. A 2004, 108, 1180911818 .

(60) Krishtalik, L. I. The mechanism of the proton transfer: an outline. Biochim. Biophys. Acta, Bioenerg. 2000, 1458, 6-27.

(61) Bell, R. P. The Tunnel Effect in Chemistry; Chapman and Hall: New York, 1980; pp 77-105.

(62) Kohen, A.; Cannlo, R.; Bartolucci, S.; Klinman, J. P. Enzyme dynamics and hydrogen tunnelling in a thermophilic alcohol dehydrogenase. Nature 1999, 399, 496-499.

(63) Ludlow, M. K.; Soudackov, A. V.; Hammes-Schiffer, S. Theoretical Analysis of the Unusual Temperature Dependence of the Kinetic Isotope Effect in Quinol Oxidation. J. Am. Chem. Soc. 2009, 131, 7094-7102.
(64) Nelsen, S. F.; Blackstock, S. C.; Kim, Y. Estimation of inner shell Marcus terms for amino nitrogen-compounds by molecular-orbital calculations. J. Am. Chem. Soc. 1987, 109, 677-682.

(65) Nelsen, S. F.; Weaver, M. N.; Pladziewicz, J. R.; Ausman, L. K.; Jentzsch, T. L.; O'Konek, J. J. Estimation of electronic coupling for intermolecular electron transfer from cross-reaction data. J. Phys. Chem. A 2006, 110, 11665-11676.

(66) For a related application of eq 12 to the calculation of $\lambda_{\mathrm{i}}$ in CEPT reactions, see refs 40 and 69 .

(67) Biner, M.; Burgi, H. B.; Ludi, A.; Rohr, C. Crystal and molecular structures of $[\mathrm{Ru}($ bpy $) 3](\mathrm{PF} 6) 3$ and $[\mathrm{Ru}(\mathrm{bpy}) 3](\mathrm{PF} 6) 2$ at $105 \mathrm{~K} . J$. Am. Chem. Soc. 1992, 114, 5197-5203.

(68) Hupp, J. T.; Weaver, M. J. Solvent, ligand and ionic charge effects on reaction entropies for simple transition-metal redox couples. Inorg. Chem. 1984, 23, 3639-3644.

(69) Markle, T. F.; Tenderholt, A. L.; Mayer, J. M. Probing Quantum and Dynamic Effects in Concerted Proton-Electron Transfer Reactions of Phenol-Base Compounds. J. Phys. Chem. B 2012, 116, 571-584.

(70) Hay, S.; Scrutton, N. S. Incorporation of hydrostatic pressure into models of hydrogen tunneling highlights a role for pressure-modulated promoting vibrations. Biochemistry 2008, 47, 9880-9887.

(71) Johannissen, L. O.; Scrutton, N. S.; Sutcliffe, M. J. How Does Pressure Affect Barrier Compression and Isotope Effects in an Enzymatic Hydrogen Tunneling Reaction? Angew. Chem., Int. Ed. 2011, 50, 2129-2132.

(72) Schmidt, M. W.; Baldridge, K. K.; Boatz, J. A.; Elbert, S. T.; Gordon, M. S.; Jensen, J. H.; Koseki, S.; Matsunga, N.; Nguyen, K. A.; $\mathrm{Su}$, S.; Windus, T. L.; Dupuis, M.; Montgomery, J. A. General atomic and molecular electronic structure system. J. Comput. Chem. 1993, 14, $1347-1363$

(73) Gordon, M. S.; Schmidt, M. W. Advances in electronic structure theory: GAMESS a decade later. In Theory and Applications of Computational Chemistry: the first forty years; Dykstra, C. E., Frenking, G., Kim, K. S., Scuseria, G. E., Eds.; Elsevier: Amsterdam, The Netherlands, 2005; pp 1167-1189.

(74) Allen, F. H.; Kennard, O.; Watson, D. G. Tables of bond lengths determined by X-ray and neutron diffraction. 1. Bond lengths in inorganic compounds. J. Chem. Soc., Perkin Trans. 2 1987, S1-S19.

(75) Bell, R. P. The Proton in Chemistry, 2nd ed.; Cornell University Press: Ithaca, NY, 1973; pp 226-249.

(76) Edwards, S. E.; Soudackov, A. V.; Hammes-Schiffer, S. Analysis of Kinetic Isotope Effects for Proton-Coupled Electron Transfer Reactions. J. Phys. Chem. A 2009, 113, 2117-2126.

(77) Pryor, W. A.; Kneipp, K. G. Primary kinetic isotope effects and nature of hydrogen-transfer transition states. Reaction of a series of free radicals with thiols. J. Am. Chem. Soc. 1971, 93, 5584-5586.

(78) Ishikawa, M.; Fukuzumi, S. Primary kinetic isotope effects on acidcatalysed reduction of p-benzoquinone derivatives by an acid-stable NADH analogue. J. Chem. Soc., Faraday Trans. 1990, 86, 3531-3536.

(79) Bronner, C.; Wenger, O. S. Kinetic Isotope Effects in Reductive Excited-State Quenching of $\mathrm{Ru}\left(2,2^{\prime}\right.$-bipyrazine $)(3)(2+)$ by Phenols. J. Phys. Chem. Lett. 2012, 3, 70-74.

(80) Nishida, Y.; Morimoto, Y.; Lee, Y.-M.; Nam, W.; Fukuzumi, S. Effects of Proton Acceptors on Formation of a Non-Heme Iron(IV)Oxo Complex via Proton-Coupled Electron Transfer. Inorg. Chem. 2013, 52, 3094-3101.

(81) Ludlow, M. K.; Soudackov, A. V.; Hammes-Schiffer, S. Electrochemical Proton-Coupled Electron Transfer of an Osmium Aquo Complex: Theoretical Analysis of Asymmetric Tafel Plots and Transfer Coefficients. J. Am. Chem. Soc. 2010, 132, 1234-1235.

(82) Horvath, S.; Fernandez, L. E.; Soudackov, A. V.; HammesSchiffer, S. Insights into proton-coupled electron transfer mechanisms of electrocatalytic H-2 oxidation and production. Proc. Natl. Acad. Sci. U. S. A. 2012, 109, 15663-15668.

(83) Liu, Y.; Liu, H.; Song, K.; Xu, Y.; Shi, Q. Theoretical Study of Proton Coupled Electron Transfer Reactions: The Effect of Hydrogen Bond Bending Motion. J. Phys. Chem. B 2015, 119, 8104-8114. 